\title{
The transition form factors for semi-leptonic weak decays of $J / \psi$ in QCD sum rules
}

\author{
Yu-Ming Wang ${ }^{1}$, Hao Zou ${ }^{1}$, Zheng-Tao $\mathrm{Wei}^{2}$, Xue-Qian $\mathrm{Li}^{2}$, and Cai-Dian Lü ${ }^{1}$ \\ ${ }^{1}$ Institute of High Energy Physics, P.O. Box 918(4), Beijing 100049, China and \\ ${ }^{2}$ Department of Physics, Nankai University, Tianjin 300071, China
}

(Dated: October 28, 2018)

\begin{abstract}
Within the Standard Model, we investigate the semi-leptonic weak decays of $J / \psi$. The various form factors of $J / \psi$ transiting to a single charmed meson $\left(D_{(d, s)}^{(*)}\right)$ are studied in the framework of the QCD sum rules. These form factors fully determine the rates of the weak semi-leptonic decays of $J / \psi$ and provide valuable information about the non-perturbative QCD effects. Our results indicate that the decay rate of the semi-leptonic weak decay mode $J / \psi \rightarrow D_{s}^{(*)-}+e^{+}+\nu_{e}$ is at order of $10^{-10}$.
\end{abstract}

PACS numbers: 13.20.Gd, 13.25.Gv, 11.55.Hx

\section{INTRODUCTION}

Although strong and electromagnetic decays of $J / \psi$ have been extensively studied for several decades, both experimental and theoretical investigations of weak decays of $J / \psi$ are much behind. Due to smallness of the strength of weak interaction, the weak decays of the $J / \psi$ are rare processes. Sanchis-Lonzano suggested to search for these rare decays whose sum of branching ratios were estimated to be at the order of $10^{-8}[1]$. Such processes hardly drew much attention because the database was far from reaching such accuracy. Thus, for a long time, few further researches on this topic were done. Thanks to the progress of accelerator and detector techniques, more accurate measurements may be carried out, thus the interest on weak decays of $J / \psi$ has been revived. The BES collaboration indeed starts to measure some rare weak decays of $J / \psi$ and eventually sets an upper bound on the branching ratio of $J / \psi \rightarrow D+e+\nu_{e}$ at order of $10^{-5}$ by using $5.8 \times 10^{7} \mathrm{~J} / \psi$ database [2]. The forthcoming upgraded BESIII can accumulate $10^{10} \mathrm{~J} / \psi$ per year [3], which makes it marginally possible to measure such weak decays of $\mathrm{J} / \psi$, at least one may expect to observe not-null such events. Thus, more careful theoretical investigation on these decays seems necessary.

Indeed, the weak decays of heavy quarkonium like $J / \psi$ offer an ideal opportunity of studying nonperturbative QCD effects, because such systems contain two heavy constituents of the same flavor. The situation is quite different from that for heavy mesons which contain only one heavy constituent, and 
the non-perturbative effects might be attributed to the light flavor, thus the heavy quark effective theory (HQET) applies. Moreover, for the weak decay of a vector meson, the polarization effect may play a role to probe the underlying dynamics and hadron structure [1] .

The weak decay of $J / \psi$ is realized via the spectator mechanism that the charm quark (antiquark) decays and the antiquark (quark) acts as a spectator. The characteristic of the decay modes is that the final state contains a single charmed hadron. The theory of weak interactions has been thoroughly investigated and the effective hamiltonian at the quark level is perfectly formulated. The main job of calculating the rates of the semi-leptonic decays of $J / \psi$ is to properly evaluate the hadronic matrix elements for $J / \psi \rightarrow D^{(*)}$, namely the transition form factors which are obviously governed by nonperturbative QCD effects. The main aim of this work is to calculate the $J / \psi \rightarrow D_{(d, s)}^{(*)}$ form factors in the QCD sum rules.

The weak decay of heavy quarkonium has been studied by virtue of heavy quark spin symmetry 1]. In that framework, the transition form factors of a heavy quarkonium to heavy pseudoscalar and vector mesons are parameterized by a universal function $\eta_{12}\left(v_{1} \cdot v_{2}\right)$ in analog to the Isgur-Wise function for the heavy meson transitions. However, the non-recoil approximation $\eta_{12}\left(v_{1} \cdot v_{2}\right) \approx 1$ was used in Ref.[1], which would bring up uncontrollable uncertainties to the estimation of decay widths. It seems helpful to re-investigate these processes based on a more rigorous theoretical framework. Motivated by the arguments, in this work we will calculate the form factors for heavy quarkonium $J / \psi$ decays into a pseudoscalar or vector meson in the QCD sum rules.

As a matter of fact, many authors have tried to evaluate the transition form factors for the heavy meson and quarkonium system in various approaches, such as the simple quark model [4], light-front approach [5], the QCD sum rules [6, 7], the perturbative QCD approach [8] and etc. The QCD sum-rule approach, which is rooted in the quantum field theory and fully relativistic, is considered to be one of the effective tools for analyzing hadronic processes [6]. Besides evaluation of hadron spectra, the QCD sum-rule technique has been applied to calculate the pion electromagnetic form factor at intermediate momentum transfer [9, 10], various weak decay channels [11, 12], the coupling constant of the strong interaction [13] and even to determine the light cone distribution amplitudes of hadrons [14]. The advantage of this method is that the non-perturbative QCD effects are included in a few parameters such as the quarkand gluon-condensates which have evident physical meaning [15].

After this introduction, we will firstly display the effective Hamiltonian relevant to the semi-leptonic decays of $J / \psi$ to $D_{d(s)}^{(*)}$, and the sum rules for form factors in section II. The Wilson coefficients of various operators which manifest the perturbative QCD effects are also calculated in this section with the help of operator product expansion (OPE) technique. The numerical analysis on the form factors are 
performed in section 【II. The decay rates of semi-leptonic decay $J / \psi \rightarrow D_{d(s)}^{(*)-} l^{+} \nu$ and a comparison of our results with that obtained based on other approaches are presented in section [V] In the last section we draw our conclusion.

\section{II. $J / \psi \rightarrow D_{d(s)}^{(*)}$ TRANSITION FORM FACTORS IN THE QCD SUM RULES}

\section{A. Definitions of $J / \psi \rightarrow D_{d(s)}^{(*)}$ transition form factors}

For the semi-leptonic decays $J / \psi \rightarrow D_{d(s)}^{(*)} l^{+} \nu_{l}$, the effective weak Hamiltonian is given by

$$
\mathcal{H}_{e f f}\left(c \rightarrow s(d) l \bar{\nu}_{l}\right)=\frac{G_{F}}{\sqrt{2}} V_{c s(d)}^{*} \bar{s}(\bar{d}) \gamma_{\mu}\left(1-\gamma_{5}\right) c \bar{\nu}_{l} \gamma^{\mu}\left(1-\gamma_{5}\right) l
$$

In order to calculate the rate of a semi-leptonic decay, the essential ingredient is the hadronic matrix element $\left\langle D_{d(s)}^{(*)}\left|\bar{s} \gamma_{\mu}\left(1-\gamma_{5}\right) c\right| J / \psi\right\rangle$ which is parameterized by various form factors [16]:

$$
\begin{aligned}
\left\langle D_{d(s)}(\right. & \left.\left.p_{2}\right)\left|\bar{q} \gamma_{\mu}\left(1-\gamma_{5}\right) c\right| J / \psi\left(\epsilon, p_{1}\right)\right\rangle \\
= & -\epsilon_{\mu \nu \alpha \beta} \epsilon^{\nu} p_{1}^{\alpha} p_{2}^{\beta} \frac{2 V\left(q^{2}\right)}{m_{\psi}+m_{D}}+i\left(m_{\psi}+m_{D}\right)\left[\epsilon_{\mu}-\frac{\epsilon \cdot q}{q^{2}} q_{\mu}\right] A_{1}\left(q^{2}\right) \\
& +i \frac{\epsilon \cdot q}{m_{\psi}+m_{D}} A_{2}\left(q^{2}\right)\left[\left(p_{1}+p_{2}\right)_{\mu}-\frac{m_{\psi}^{2}-m_{D}^{2}}{q^{2}} q_{\mu}\right]+2 i m_{\psi} \frac{\epsilon \cdot q}{q^{2}} q_{\mu} A_{0}\left(q^{2}\right), \\
\left\langle D_{d(s)}^{*}\left(\epsilon_{2}, p_{2}\right)\left|\bar{q} \gamma_{\mu}\left(1-\gamma_{5}\right) c\right| J / \psi\left(\epsilon_{1}, p_{1}\right)\right\rangle & \\
= & -i \epsilon_{\mu \nu \alpha \beta} \epsilon_{1}^{\alpha} \epsilon_{2}^{* \beta}\left[\left(p_{1}^{\nu}+p_{2}^{\nu}-\frac{m_{\psi}^{2}-m_{D^{*}}^{2}}{q^{2}} q^{\nu}\right) \tilde{A}_{1}\left(q^{2}\right)+\frac{m_{\psi}^{2}-m_{D^{*}}^{2}}{q^{2}} q^{\nu} \tilde{A}_{2}\left(q^{2}\right)\right] \\
& +\frac{i}{m_{\psi}^{2}-m_{D^{*}}^{2}} \epsilon_{\mu \nu \alpha \beta} p_{1}^{\alpha} p_{2}^{\beta}\left[\tilde{A}_{3}\left(q^{2}\right) \epsilon_{1}^{\nu} \epsilon_{2}^{*} \cdot q-\tilde{A}_{4}\left(q^{2}\right) \epsilon_{2}^{* \nu} \epsilon_{1} \cdot q\right] \\
& +\left(\epsilon_{1} \cdot \epsilon_{2}^{*}\right)\left[-\left(p_{1 \mu}+p_{2 \mu}\right) \tilde{V}_{1}\left(q^{2}\right)+q_{\mu} \tilde{V}_{2}\left(q^{2}\right)\right] \\
& +\frac{\left(\epsilon_{1} \cdot q\right)\left(\epsilon_{2}^{*} \cdot q\right)}{m_{\psi}^{2}-m_{D^{*}}^{2}}\left[\left(p_{1 \mu}+p_{2 \mu}-\frac{m_{\psi}^{2}-m_{D^{*}}^{2}}{q^{2}} q_{\mu}\right) \tilde{V}_{3}\left(q^{2}\right)\right. \\
& \left.+\frac{m_{\psi}^{2}-m_{D^{*}}^{2}}{q^{2}} q_{\mu} \tilde{V}_{4}\left(q^{2}\right)\right]-\left(\epsilon_{1} \cdot q\right) \epsilon_{2}^{*} \tilde{V}_{5}\left(q^{2}\right)+\left(\epsilon_{2}^{*} \cdot q\right) \epsilon_{1 \mu} \tilde{V}_{6}\left(q^{2}\right),
\end{aligned}
$$

where the convention $\operatorname{Tr}\left[\gamma_{\mu} \gamma_{\nu} \gamma_{\rho} \gamma_{\sigma} \gamma_{5}\right]=4 i \epsilon_{\mu \nu \rho \sigma}$ is adopted. For a transition of $J / \psi$ into a charmed pseudoscalar meson which is induced by the weak current, there are four independent form factors: $V, A_{0}, A_{1}, A_{2}$; while there are ten form factors for $J / \psi$ transiting to a charmed vector meson which are parameterized as $\tilde{A}_{i}(i=1,2,3,4), \tilde{V}_{j}(j=1,2,3,4,5,6)$. It is worthwhile to emphasize that the parametrization of the hadronic matrix element for $J / \psi$ to vector meson given in Eq. (3) is less studied before. Similar matrix element for a transition of a vector to another vector meson which is induced by the electromagnetic current was investigated by Kagan in Ref. [17]. 


\section{B. The transition form factors in the QCD sum rules}

In this subsection, we calculate transition form factors of $J / \psi \rightarrow D_{(d, s)}^{(*)-}$ in the QCD sum rules. Here we present the formulations for $J / \psi \rightarrow D_{s}^{(*)-}$ transition explicitly, while the expressions for $J / \psi \rightarrow D^{(*)-}$ can be obtained by simple replacements of $D_{s}^{(*)-} \rightarrow D^{(*)-}$ and $s$ quark to $d$ quark.

\section{The matrix element for $J / \psi \rightarrow D_{s}^{-}$}

Following the standard procedure of the QCD sum rules [9], we write the three-point correlation function for $J / \psi$ to $D_{s}^{-}$as

$$
\Pi_{\mu \nu}=i^{2} \int d^{4} x d^{4} y e^{-i p_{1} \cdot y+i p_{2} \cdot x}\left\langle 0\left|j_{5}^{D_{s}^{-}}(x) j_{\mu}(0) j_{\nu}^{J / \psi}(y)\right| 0\right\rangle,
$$

where the current $j_{\nu}^{J / \psi}(y)=\bar{c}(y) \gamma_{\nu} c(y)$ represents the $J / \psi$ channel; $j_{\mu}(0)=\bar{s} \gamma_{\mu}\left(1-\gamma_{5}\right) c$ is the weak current and $j_{5}^{D^{-}}(x)=\bar{c}(x) i \gamma_{5} s(x)$ corresponds to the $D_{s}^{-}$channel. In terms of the following definitions,

$$
\left\langle 0\left|\bar{c} \gamma_{\nu} c\right| J / \psi\right\rangle=m_{\psi} f_{\psi} \epsilon_{\nu}^{\lambda}, \quad\left\langle 0\left|\bar{c} i \gamma_{5} s\right| D_{s}\right\rangle=\frac{f_{D_{s}} m_{D_{s}}^{2}}{m_{c}+m_{s}}
$$

we can insert a complete set of hadronic states with the quantum numbers the same as $J / \psi$ and $D_{s}^{-}$to achieve the hadronic representation of the correlator (4)

$$
\Pi_{\mu \nu}=\frac{f_{D_{s}} m_{D_{s}}^{2}\left\langle D_{s}\left|j_{\mu}\right| J / \psi\right\rangle m_{\psi} f_{\psi} \epsilon_{\nu}^{* \lambda}}{\left(m_{J / \psi}^{2}-p_{1}^{2}\right)\left(m_{D_{s}}^{2}-p_{2}^{2}\right)\left(m_{c}+m_{s}\right)}+\text { contributions from higher states. }
$$

Obviously the concerned lowest hadronic states are $J / \psi$ and $D_{s}$, while the terms with "higher states" represent contributions coming from higher excited states and continuum. Using the double dispersion relation, the contributions of excited states and continuum can be expressed as

$$
\text { contributions from higher states }=\iint_{\sum_{12}} d s_{1} d s_{2} \frac{\rho_{\mu \nu}^{h}\left(s_{1}, s_{2}, q^{2}\right)}{\left(s_{1}-p_{1}^{2}\right)\left(s_{2}-p_{2}^{2}\right)}+\text { subtraction terms, }
$$

where $\sum_{12}$ denotes the integration region in the $\left(s_{1}, s_{2}\right)$ plane. $\rho_{\mu \nu}^{h}$ is the spectral density at the hadron level. The subtraction terms are polynomials of either $p_{1}$ or $p_{2}$, which should disappear after performing the double Borel transformation $\hat{\mathcal{B}}_{M_{1}^{2}} \hat{\mathcal{B}}_{M_{2}^{2}}$, with

$$
\hat{\mathcal{B}}_{M_{i}^{2}}=\lim _{\substack{-p_{i}^{2}, n \rightarrow \infty \\-p_{i}^{2} / n=M^{2}}} \frac{\left(-p_{i}^{2}\right)^{(n+1)}}{n !}\left(\frac{d}{d p_{i}^{2}}\right)^{n}
$$

On the other side, we calculate the correlation function at the quark level by using the OPE as

$$
\Pi_{\mu \nu}=-f_{0} \epsilon_{\mu \nu \alpha \beta} p_{1}^{\alpha} p_{2}^{\beta}-i\left(f_{1} p_{1_{\mu}} p_{1_{\nu}}+f_{2} p_{2 \mu} p_{2_{\nu}}+f_{3} p_{2 \mu} p_{1_{\nu}}+f_{4} p_{1_{\mu}} p_{2_{\nu}}+f_{5} g_{\mu \nu}\right),
$$


where each coefficient contains contributions from both perturbative part and the non-perturbative part whose effects manifest in several typical condensates,

$$
f_{i}=f_{i}^{p e r t} \mathbf{I}+f_{i}^{q q}\langle\bar{q} q\rangle+f_{i}^{G G}\langle G G\rangle+f_{i}^{q G q}\langle\bar{q} G q\rangle+\ldots
$$

with $f_{i}^{p e r t}, f_{i}^{q q}, f_{i}^{G G}, f_{i}^{q G q}, \ldots$ denoting the contributions to the correlation functions from dimension 0,3 , $4,5, \ldots$ operators. By the quark-hadron duality, one may match the two different representations of the correlation function and perform the double Borel transformation on variables $p_{1}$ and $p_{2}$, then we get the sum rules for the form factors

$$
\begin{aligned}
V\left(q^{2}\right) & =-\frac{\left(m_{c}+m_{s}\right)\left(m_{\psi}+m_{D_{s}}\right)}{2 m_{\psi} f_{\psi} f_{D_{s}} m_{D_{s}}^{2}} e^{m_{\psi}^{2} / M_{1}^{2}} e^{m_{D_{s}}^{2} / M_{2}^{2}} M_{1}^{2} M_{2}^{2} \hat{\mathcal{B}} f_{0}, \\
A_{1}\left(q^{2}\right) & =\frac{\left(m_{c}+m_{s}\right)}{\left(m_{\psi}+m_{D_{s}}\right) m_{\psi} f_{\psi} f_{D_{s}} m_{D_{s}}^{2}} e^{m_{\psi}^{2} / M_{1}^{2}} e^{m_{D_{s}}^{2} / M_{2}^{2}} M_{1}^{2} M_{2}^{2} \hat{\mathcal{B}} f_{5}, \\
A_{2}\left(q^{2}\right) & =-\frac{\left(m_{c}+m_{s}\right)\left(m_{\psi}+m_{D_{s}}\right)}{2 m_{\psi} f_{\psi} f_{D_{s}} m_{D_{s}}^{2}} e^{m_{\psi}^{2} / M_{1}^{2}} e^{m_{D_{s}}^{2} / M_{2}^{2}} M_{1}^{2} M_{2}^{2} \hat{\mathcal{B}}\left(f_{2}+f_{4}\right), \\
A_{0}\left(q^{2}\right) & =-\frac{\left(m_{c}+m_{s}\right)}{2 m_{\psi}^{2} f_{\psi} f_{D_{s}} m_{D_{s}}^{2}} e^{m_{\psi}^{2} / M_{1}^{2}} e^{m_{D_{s}}^{2} / M_{2}^{2}} M_{1}^{2} M_{2}^{2}\left[\hat{\mathcal{B}}\left(f_{2}+f_{4}\right) \frac{m_{\psi}^{2}-m_{D_{s}}^{2}}{2}-\hat{\mathcal{B}}\left(f_{2}-f_{4}\right) \frac{q^{2}}{2}-\hat{\mathcal{B}} f_{5}\right] .
\end{aligned}
$$

\section{The matrix element for $J / \psi \rightarrow D_{s}^{*-}$}

The three-point correlation function of $J / \psi$ to $D_{s}^{*-}$ is

$$
\Pi_{\mu \nu \rho}=i^{2} \int d^{4} x d^{4} y e^{-i p_{1} \cdot y+i p_{2} \cdot x}\left\langle 0\left|j_{\rho}^{D_{s}^{*}}(x) j_{\mu}(0) j_{\nu}^{J / \psi}(y)\right| 0\right\rangle,
$$

where the current $j_{\rho}^{D_{s}^{*}}(x)=\bar{c}(x) \gamma_{\rho} s(x)$ denotes the $D_{s}^{*-}$ channel, and $j_{\nu}^{J / \psi}(y), j_{\mu}(0)$ are defined as in the above subsection. One the one hand, inserting the hadron states, the correlation function is written as

$$
\Pi_{\mu \nu \rho}=\frac{m_{D_{s}^{*}} f_{D_{s}^{*}} \epsilon_{\rho}^{\prime \lambda^{\prime}}\left\langle D_{s}^{*}\left|j_{\mu}\right| J / \psi\right\rangle m_{J / \psi} f_{J / \psi} \epsilon_{\nu}^{* \lambda}}{\left(m_{J / \psi}^{2}-p_{1}^{2}\right)\left(m_{D_{s}^{*}}^{2}-p_{2}^{2}\right)}+\iint d s_{1} d s_{2} \frac{\rho_{\mu \nu \rho}^{h}\left(s_{1}, s_{2}, q^{2}\right)}{\left(s_{1}-p_{1}^{2}\right)\left(s_{2}-p_{2}^{2}\right)}+\text { subtraction terms. }
$$

On the other hand, the correlation function at the quark level is formulated as

$$
\begin{aligned}
& \Pi_{\mu \nu \rho}=i F_{1} \epsilon_{\mu \nu \alpha \beta} p_{1}^{\alpha} p_{2}^{\beta} p_{1 \rho}+i F_{2} \epsilon_{\mu \nu \alpha \beta} p_{1}^{\alpha} p_{2}^{\beta} p_{2 \rho}+i F_{3} \epsilon_{\mu \rho \alpha \beta} p_{1}^{\alpha} p_{2}^{\beta} p_{1_{\nu}}+i F_{4} \epsilon_{\mu \rho \alpha \beta} p_{1}^{\alpha} p_{2}^{\beta} p_{2_{\nu}} \\
& \quad+i F_{5} \epsilon_{\nu \rho \alpha \beta} p_{1}^{\alpha} p_{2}^{\beta} p_{1 \mu}+i F_{6} \epsilon_{\nu \rho \alpha \beta} p_{1}^{\alpha} p_{2}^{\beta} p_{2 \mu}+F_{7} g_{\mu \nu} p_{1 \rho}+F_{8} g_{\mu \rho} p_{1_{\nu}}+F_{9} g_{\nu \rho} p_{1 \mu}+F_{10} g_{\mu \nu} p_{2 \rho} \\
& \quad+F_{11} g_{\mu \rho} p_{2 \nu}+F_{12} g_{\nu \rho} p_{2 \mu}+F_{13} p_{1 \mu} p_{1 \nu} p_{1 \rho}+F_{14} p_{2 \mu} p_{2 \nu} p_{1 \rho}+F_{15} p_{1 \mu} p_{2 \nu} p_{1 \rho}+F_{16} p_{2 \mu} p_{1_{\nu}} p_{1 \rho} \\
& \quad+F_{17} p_{2 \mu} p_{2 \nu} p_{2 \rho}+F_{18} p_{1 \mu} p_{1 \nu} p_{2 \rho}+F_{19} p_{2 \mu} p_{1 \nu} p_{2 \rho}+F_{20} p_{1 \mu} p_{2_{\nu}} p_{1_{\rho} \rho},
\end{aligned}
$$

where each coefficient $F_{i}$ includes contributions from both perturbative and nonperturbative parts, and is written explicitly as

$$
F_{i}=F_{i}^{p e r t} \mathbf{I}+F_{i}^{q q}\langle\bar{q} q\rangle+F_{i}^{G G}\langle G G\rangle+F_{i}^{q G q}\langle\bar{q} G q\rangle+\ldots
$$


Again, equating the correlation functions calculated in these two frameworks and performing the Borel transformations on both sides, we derive the form factors of $J / \psi \rightarrow D_{s}^{*-}$ as

$$
\begin{aligned}
& \tilde{A}_{1}\left(q^{2}\right)=-\frac{1}{4 m_{\psi} f_{\psi} m_{D^{*}-} f_{D_{s}^{*}}} e^{m_{\psi}^{2} / M_{1}^{2}} e^{m_{D_{s}^{*}}^{2} / M_{2}^{2}} M_{1}^{2} M_{2}^{2} \hat{\mathcal{B}}\left[\left(F_{5}-F_{6}\right) q^{2}+\left(F_{5}+F_{6}\right)\left(m_{\psi}^{2}-m_{D_{s}^{*}}^{2}\right)\right], \\
& \tilde{A}_{2}\left(q^{2}\right)=\frac{m_{D_{s}^{*}}^{4}-2\left(q^{2}+m_{\psi}^{2}\right) m_{D_{s}^{*}}^{2}+\left(q^{2}-m_{\psi}^{2}\right)^{2}}{4\left(m_{D_{s}^{*}}^{2}-m_{\psi}^{2}\right) m_{\psi} f_{\psi} m_{D_{s}^{*}} f_{D_{s}^{*}}} e^{m_{\psi}^{2} / M_{1}^{2}} e^{m_{D_{s}^{*}}^{2} / M_{2}^{2}} M_{1}^{2} M_{2}^{2} \hat{\mathcal{B}}\left(F_{5}+F_{6}\right), \\
& \tilde{A}_{3}\left(q^{2}\right)=\frac{m_{\psi}^{2}-m_{D_{s}^{*}}^{2}}{m_{\psi} f_{\psi} m_{D_{s}^{*}} f_{D_{s}^{*}}} e^{m_{\psi}^{2} / M_{1}^{2}} e^{m_{D_{s}^{*}}^{2} / M_{2}^{2}} M_{1}^{2} M_{2}^{2} \hat{\mathcal{B}}\left(F_{1}-F_{5}\right), \\
& \tilde{A}_{4}\left(q^{2}\right)=\frac{m_{\psi}^{2}-m_{D_{s}^{*}}^{2}}{m_{\psi} f_{\psi} m_{D_{s}^{*}} f_{D_{s}^{*}}} e^{m_{\psi}^{2} / M_{1}^{2}} e^{m_{D_{s}^{*}}^{2} / M_{2}^{2}} M_{1}^{2} M_{2}^{2} \hat{\mathcal{B}}\left(F_{4}+F_{6}\right), \\
& \tilde{V}_{1}\left(q^{2}\right)=-\frac{1}{2 m_{\psi} f_{\psi} m_{D_{s}^{*}} f_{D_{s}^{*}}} e^{m_{\psi}^{2} / M_{1}^{2}} e^{m_{D_{s}^{*}}^{2} / M_{2}^{2}} M_{1}^{2} M_{2}^{2} \hat{\mathcal{B}}\left(F_{9}+F_{12}\right), \\
& \tilde{V}_{2}\left(q^{2}\right)=\frac{1}{2 m_{\psi} f_{\psi} m_{D_{s}^{*}} f_{D_{s}^{*}}} e^{m_{\psi}^{2} / M_{1}^{2}} e^{m_{D_{s}^{*}}^{2} / M_{2}^{2}} M_{1}^{2} M_{2}^{2} \hat{\mathcal{B}}\left(F_{9}-F_{12}\right), \\
& \tilde{V}_{3}\left(q^{2}\right)=\frac{m_{D_{s}^{*}}^{2}-m_{\psi}^{2}}{2 m_{\psi} f_{\psi} m_{D_{s}^{*}} f_{D_{s}^{*}}} e^{m_{\psi}^{2} / M_{1}^{2}} e^{m_{D_{s}^{*}}^{2} / M_{2}^{2}} M_{1}^{2} M_{2}^{2} \hat{\mathcal{B}}\left(F_{14}+F_{15}\right), \\
& \tilde{V}_{4}\left(q^{2}\right)=\frac{1}{2 m_{\psi} f_{\psi} m_{D_{s}^{*}} f_{D_{s}^{*}}} e^{m_{\psi}^{2} / M_{1}^{2}} e^{m_{D_{s}^{*}}^{2} / M_{2}^{2}} M_{1}^{2} M_{2}^{2} \hat{\mathcal{B}}\left[\left(F_{14}-F_{15}\right) q^{2}+\left(F_{14}+F_{15}\right)\left(m_{D_{s}^{*}}^{2}-m_{\psi}^{2}\right)\right], \\
& \tilde{V}_{5}\left(q^{2}\right)=\frac{1}{m_{\psi} f_{\psi} m_{D_{s}^{*}} f_{D_{s}^{*}}} e^{m_{\psi}^{2} / M_{1}^{2}} e^{m_{D_{s}^{*}}^{2} / M_{2}^{2}} M_{1}^{2} M_{2}^{2} \hat{\mathcal{B}} F_{11}, \\
& \tilde{V}_{6}\left(q^{2}\right)=\frac{1}{m_{\psi} f_{\psi} m_{D^{*-}} f_{D_{s}^{*}}} e^{m_{\psi}^{2} / M_{1}^{2}} e^{m_{D_{s}^{*}}^{2} / M_{2}^{2}} M_{1}^{2} M_{2}^{2} \hat{\mathcal{B}} F_{7}
\end{aligned}
$$

\section{The Wilson coefficients for correlation function $\Pi_{\mu \nu}$}

In this subsection we calculate the Wilson coefficients which are defined above. To guarantee sufficient theoretical accuracy, the correlation functions are required to be expanded up to dimension-5 operators, namely quark-gluon mixing condensate. The dimension-6 operators, such as the four quark condensates, are small and further suppressed by $O\left(\alpha_{s}^{2}\right)$, so can be safely neglected in our calculations.

The diagrams which depict the contributions from the perturbative part and nonperturbative condensates are shown in Fig. 1. The first diagram results in the Wilson coefficient of the unit operator; the second diagram is relevant to the contribution of quark condensate, where the heavy-quark condensate is neglected. The Wilson coefficient of the two-gluon condensate operator is obtained from Fig. 1 (c-h). The last two diagrams Fig. 1(i-j) stand for the contribution of quark-gluon mixing condensate. In this work, all of the Wilson coefficients are calculated at the lowest order in the running coupling constant of strong interaction. 


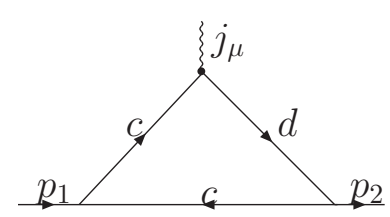

(a)

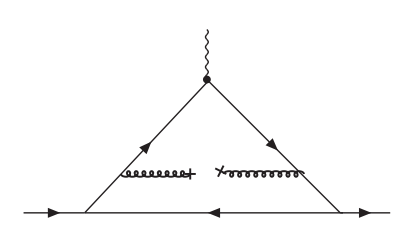

$(d)$
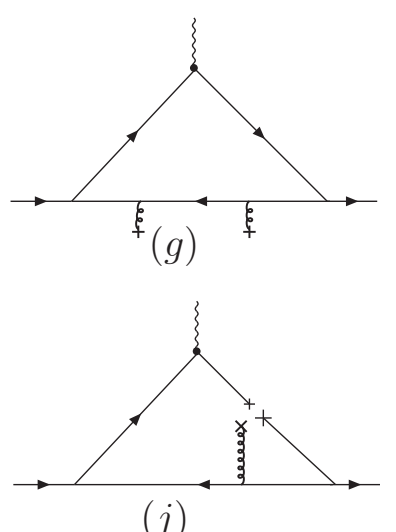

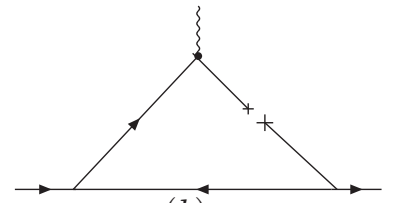

$(b)$

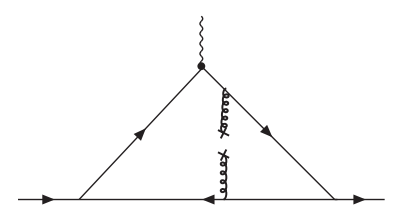

(e)

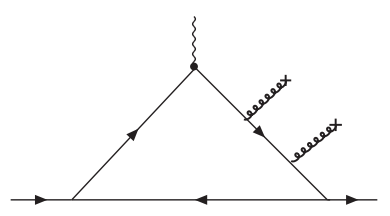

$(h)$

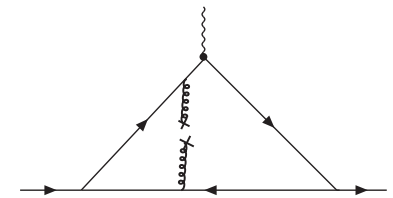

$(c)$

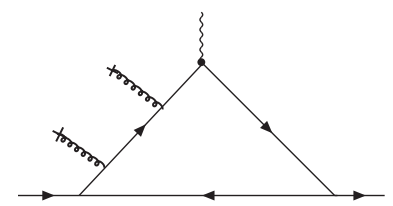

$(f)$

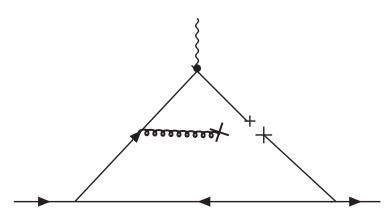

$(i)$

FIG. 1: Graphs for the Wilson coefficients in the operator product expansion of the correlation function. (a) is for the contribution of unit operator; (b) for the two-quark condensate; (c-h) describe the contributions from gluon condensate, (i-j) is for the quark-gluon mixing condensate.

\section{Perturbative contributions to Wilson coefficients for $\Pi_{\mu \nu}$}

The perturbative contribution to the three-point correlation function $\Pi_{\mu \nu}$ shown in Fig. 10 (a) is included in the following amplitude

$$
C_{\mu \nu}^{\text {pert }}=3 i^{2} \int \frac{d^{4} k}{(2 \pi)^{4}}(-1) \operatorname{Tr}\left[\gamma_{\nu} \frac{i}{\not k-m_{c}} i \gamma_{5} \frac{i}{\not p_{2}+\not k-m_{q}} \gamma_{\mu}\left(1-\gamma_{5}\right) \frac{i}{\not p_{1}+\not k-m_{c}}\right]
$$

where $m_{q}$ denotes the mass of the light quark in the $D$ meson, and the factor " 3 " is due to the color loop. Using the dispersion relation, $C_{0 \mu \nu}^{\text {pert }}$ is written as

$$
C_{0 \mu \nu}^{p e r t}=\iint d s_{1} d s_{2} \frac{\rho_{\mu \nu}^{\text {pert }}\left(s_{1}, s_{2}, q^{2}\right)}{\left(s_{1}-p_{1}^{2}\right)\left(s_{2}-p_{2}^{2}\right)} .
$$


The integration region is determined by the following condition

$$
-1 \leq \frac{2 s_{1}\left(s_{2}+m_{c}^{2}-m_{q}^{2}\right)-s_{1}\left(s_{1}+s_{2}-q^{2}\right)}{\lambda^{1 / 2}\left(s_{1}, s_{2}, q^{2}\right) \lambda^{1 / 2}\left(m_{c}^{2}, s_{1}, m_{c}^{2}\right)} \leq 1,
$$

where $\lambda(a, b, c)=a^{2}+b^{2}+c^{2}-2 a b-2 a c-2 b c$. The standard way to calculate the spectral function $\rho_{\mu \nu}\left(s_{1}, s_{2}, q^{2}\right)$ is described below [9]: Firstly, it is essential to calculate the double discontinuity of the amplitude, which can be realized by putting all the internal quark lines of Fig. 1 (a) on their mass-shell and substituting the denominators of the quark propagators by the $\delta$ functions based on the Cutkosky's cutting rule,

$$
\frac{1}{k^{2}-m^{2}+i \epsilon} \rightarrow-2 \pi i \delta\left(k^{2}-m^{2}\right)
$$

Then, the spectral function can be easily achieved. Finally, we get the expression of the spectral function in the following form

$$
\begin{aligned}
\rho_{\mu \nu}\left(p_{1}^{2}, p_{2}^{2}, q^{2}\right)= & \frac{3}{(2 \pi i)^{2}}(-2 \pi i)^{3} \int \frac{d^{4} k}{(2 \pi)^{4}} \operatorname{Tr}\left[\gamma_{\nu}\left(\not k+m_{c}\right) \gamma_{5}\left(\not p_{2}+\not k+m_{q}\right) \gamma_{\mu}\left(1-\gamma_{5}\right)\left(\not p_{1}+\not k+m_{c}\right)\right] \\
& \delta\left(k^{2}-m_{c}^{2}\right) \delta\left[\left(p_{2}+k\right)^{2}-m_{q}^{2}\right] \delta\left[\left(p_{1}-k\right)^{2}-m_{c}^{2}\right] .
\end{aligned}
$$

After tedious calculations, one finally obtains the perturbative contribution to the correlation function, which can be decomposed as the sum of various terms according to different Lorentz structures, namely,

$$
\rho_{\mu \nu}^{\text {pert }}=-\rho_{0}^{\text {pert }} \epsilon_{\mu \nu \alpha \beta} p_{1}^{\alpha} p_{2}^{\beta}-i\left(\rho_{1}^{\text {pert }} p_{1_{\mu}} p_{1_{\nu}}+\rho_{2}^{\text {pert }} p_{2 \mu} p_{2 \nu}+\rho_{3}^{\text {pert }} p_{2 \mu} p_{1_{\nu}}+\rho_{4}^{\text {pert }} p_{1_{\mu}} p_{2 \nu}+\rho_{5}^{\text {pert }} g_{\mu \nu}\right)
$$

The expressions for the $\rho_{i}^{\text {pert }}$ are a bit more tedious, so that we will display their explicit forms in Appendix A.

\section{The quark condensate contribution}

Now we turn to calculating the Wilson coefficient of the quark condensate operator, which are shown in Fig. 1 (b). One can easily find that it does not contribute to the correlation function after performing the double Borel transformation on both variables $p_{1}^{2}$ and $p_{2}^{2}$, since the propagator of this diagram $\frac{1}{\left(p_{2}^{2}-m_{c}^{2}\right)\left(q^{2}-m_{c}^{2}\right)}$ only depends on variable $p_{2}^{2}$. In other words, the Wilson coefficient of dimension-3 two quark condensate turns to be zero in the leading order of heavy quark mass expansion after carrying out the double Borel transformation. As can be seen, vanishing of the contributions from quark condensate is independent on the structures of the effective vertices, therefore, it also does not contribute to the decays of $J / \psi$ into a vector meson for the same reason. Below, we do not need to investigate the contributions of quark condensate to $J / \psi \rightarrow D^{*}$ based on this argument. 


\section{The contribution from gluon condensate}

The diagrams which determine the Wilson coefficient of the gluon condensate are shown in Fig. 1 (c-h). The standard way is using the so-called fixed -point gauge technique. The gauge fixing condition is

$$
x^{\mu} A_{\mu}^{a}=0,
$$

where $A_{\mu}^{a}$ is the gluon field. In the momentum space, $A_{\mu}^{a}(k)$ is transformed to the gauge invariant field strength as

$$
A_{\mu}^{a}(k)=-\frac{i}{2}(2 \pi)^{4} G_{\rho \mu}^{a}(0) \frac{\partial}{\partial k_{\rho}} \delta^{4}(k)+\ldots
$$

Indeed, the loop integral

$$
I_{\mu_{1}, \mu_{2}, \ldots \mu_{n}}(a, b, c)=\int \frac{d^{4} k}{(2 \pi)^{4}} \frac{k_{\mu_{1}} k_{\mu_{2}} \ldots k_{\mu_{n}}}{\left[k^{2}-m^{2}\right]^{a}\left[\left(p_{1}+k\right)^{2}-m_{1}^{2}\right]^{b}\left[\left(p_{2}+k\right)^{2}-m_{2}^{2}\right]^{c}},
$$

which is encountered in the work, is not easy to be performed by the Feynman parameter method. One alternative way to calculate this kind of integrals has been extensively discussed in the Ref. [15, 18, 19, 20], where the authors suggested to work in the Euclidean space-time and employ the Schwinger representation for propagators. Instead, in our work, we follow the method employed in Ref. [21, 22], namely, directly calculate the imaginary part of the integrals in terms of the Cutkosky's rule.

With the help of the Mathematical package "FeynCalc", we finally get the contributions of Fig. 1 (c-h) at the price of some long and tedious derivations and time-consuming computer computations. The contributions of the gluon-condensates from various sources cancel each other completely after carrying out the double Borel transformation to the variables $p_{1}^{2}$ and $p_{2}^{2}$. Therefore the diagrams involving the gluon condensate do not contribute to the transition of vector meson $J / \psi$ to a pseudoscalar $D$ meson. This argument also applies to the transition of a pseudoscalar meson to a vector which was discussed in Ref. [21, 22], since topologies of the Feynman diagrams which result in the Wilson coefficient of the gluon condensate are the same. As analyzed later, it is also true for the transition of $J / \psi$ to a vector meson. However, we find that the flavor-changing neutral current process can receive non-zero contributions from the gluon condensate. It should be noted that the null contributions of gluon condensates to sum rules for the weak transition $c \rightarrow s(d)$ are different from that obtained in Ref. [18, 19, 20], where the method they adopted does not allow for the substraction of continuum contributions. 


\section{The quark-gluon mixing condensate contribution}

Finally, we go on calculating the Wilson coefficients of the dimension-5 operator $\langle\bar{q} G q\rangle$. Only two diagrams shown in Fig. 1 (i-j) are involved. Concentrating on these two diagrams, we find that they do not contribute to the correlation function, due to the same reason as that for the null contribution from quark condensate, namely, only the variable $p_{2}^{2}$ appears in the propagators, the amplitude will vanish due to the double Borel transformation.

As mentioned at the beginning of this section, we do not consider the four quark condensate, hence only the perturbative part which corresponds to Fig.1 (a), offers a non-zero contribution to the correlation function.

\section{The Wilson coefficients for the operators contributing to the correlation function $\Pi_{\mu \nu \rho}$}

After above lengthy discussions, a computation of the correlation function $\Pi_{\mu \nu \rho}$ which determines the transition amplitude of $J / \psi$ to a vector meson is straightforward. Repeating the previous calculations but replacing the vertex for the pseudoscalar meson to that for a vector meson, one can obtain the expressions of the Wilson coefficients for all the concerned operators.

\section{The calculations of the perturbative contribution to $\Pi_{\mu \nu \rho}$}

The Wilson coefficient of the perturbative part corresponding to Fig. 1 (a) is

$$
C_{\mu \nu \rho}^{\text {pert }}=3 i^{2} \int \frac{d^{4} k}{(2 \pi)^{4}}(-1) \operatorname{Tr}\left[\gamma_{\nu} \frac{i}{\not k-m_{c}} \gamma_{\rho} \frac{i}{\not p_{2}+\not k-m_{d}} \gamma_{\mu}\left(1-\gamma_{5}\right) \frac{i}{\not p_{1}+\not k-m_{c}}\right] .
$$

We rewrite it in the form of dispersion integrals for the sake of connecting it to the hadronic spectral density based on the assumption of the quark-hadron duality, as

$$
C_{\mu \nu \rho}^{p e r t}=\iint d s_{1} d s_{2} \frac{\rho_{\mu \nu \rho}^{p e r t}\left(s_{1}, s_{2}, q^{2}\right)}{\left(s_{1}-p_{1}^{2}\right)\left(s_{2}-p_{2}^{2}\right)}
$$

The integration region is the same as that for the $C_{\mu \nu}^{p e r t}$, which is presented in Eq. (31). Setting all the internal quark lines on their mass shells, we derive the spectral function $\rho_{\mu \nu \rho}^{\text {pert }}$ as

$$
\begin{aligned}
& \rho_{\mu \nu \rho}^{\text {pert }}=i \rho_{1}^{\text {pert }} \epsilon_{\mu \nu \alpha \beta} p_{1}^{\alpha} p_{2}^{\beta} p_{1 \rho}+i \rho_{2}^{\prime \text { pert }} \epsilon_{\mu \nu \alpha \beta} p_{1}^{\alpha} p_{2}^{\beta} p_{2 \rho}+i \rho_{3}^{\prime \text { pert }} \epsilon_{\mu \rho \alpha \beta} p_{1}^{\alpha} p_{2}^{\beta} p_{1 \nu}+i \rho_{4}^{\prime \text { pert }} \epsilon_{\mu \rho \alpha \beta} p_{1}^{\alpha} p_{2}^{\beta} p_{2 \nu} \\
& +i \rho_{5}^{\prime \text { pert }} \epsilon_{\nu \rho \alpha \beta} p_{1}^{\alpha} p_{2}^{\beta} p_{1_{\mu}}+i \rho_{6}^{\prime \text { pert }} \epsilon_{\nu \rho \alpha \beta} p_{1}^{\alpha} p_{2}^{\beta} p_{2 \mu}+\rho_{7}^{\prime \text { pert }} g_{\mu \nu} p_{1_{\rho}}+\rho_{8}^{\prime \text { pert }} g_{\mu \rho} p_{1_{\nu}}+\rho_{9}^{\prime \text { pert }} g_{\nu \rho} p_{1_{\mu}} \\
& +\rho_{10}^{\prime \text { pert }} g_{\mu \nu} p_{2 \rho}+\rho_{11}^{\prime \text { pert }} g_{\mu \rho} p_{2 \nu}+\rho_{12}^{\text {pert }} g_{\nu \rho} p_{2 \mu}+\rho_{13}^{\prime \text { pert }} p_{1 \mu} p_{1 \nu} p_{1 \rho}+\rho_{14}^{\prime \text { pert }} p_{2 \mu} p_{2 \nu} p_{1 \rho}+\rho_{15}^{\prime \text { pert }} p_{1 \mu} p_{1 \nu} p_{1 \rho} \\
& +\rho_{16}^{\prime \text { pert }} p_{2 \mu} p_{1 \nu} p_{1 \rho}+\rho_{17}^{\prime \text { pert }} p_{2 \mu} p_{2 \nu} p_{2 \rho}+\rho_{18}^{\prime \text { pert }} p_{1_{\mu}} p_{1 \nu} p_{2 \rho}+\rho_{19}^{\prime \text { pert }} p_{2 \mu} p_{1_{\nu}} p_{2 \rho}+\rho_{20}^{\prime \text { pert }} p_{1_{\mu}} p_{2 \nu} p_{1 \rho} \text {. }
\end{aligned}
$$


Only $\rho_{i}^{\prime \text { pert }}(i=1,4,5,6,7,9,11,12,14,15)$ are related to the form factors $\tilde{V}_{1}, \tilde{V}_{2}, \tilde{V}_{3}, \tilde{V}_{4}, \tilde{V}_{5}, \tilde{V}_{6}, \tilde{A}_{1}, \tilde{A}_{2}$, $\tilde{A}_{3}, \tilde{A}_{4}$, and we display their expressions in Appendix B.

\section{The contribution of gluon condensate to $\Pi_{\mu \nu \rho}$}

Similar to the derivation made above, we easily obtain the Wilson coefficient of the gluon condensate which may contribute to the correlation function $\Pi_{\mu \nu \rho}$. Then we rewrite the Wilson coefficient in the form of dispersion integrals:

$$
C_{\mu \nu \rho}^{G G}=\iint d s_{1} d s_{2} \frac{\rho_{\mu \nu \rho}^{G G}\left(s_{1}, s_{2}, q^{2}\right)}{\left(s_{1}-p_{1}^{2}\right)\left(s_{2}-p_{2}^{2}\right)},
$$

where the integral region is the same as that for the perturbative part.

The Lorentz structures corresponding to $\rho_{i}^{(G G)}(i=1,4,5,6,7,9,11,12,14,15)$ are

$$
\begin{aligned}
\rho_{\mu \nu \rho}^{G G}= & i \rho_{1}^{\prime G G} \epsilon_{\mu \nu \rho \lambda} p_{1}^{\lambda}+i \rho_{4}^{\prime G G} \epsilon_{\mu \nu \rho \lambda} p_{2}^{\lambda}+i \rho_{5}^{\prime G G} \epsilon_{\mu \nu \alpha \beta} p_{1}^{\alpha} p_{1}^{\beta} p_{1_{\nu}}+i \rho_{6}^{\prime G G} \epsilon_{\mu \rho \alpha \beta} p_{1}^{\alpha} p_{1}^{\beta} p_{1 \rho}+\rho_{7}^{\prime G G} g_{\mu \nu} p_{1 \rho} \\
& +\rho_{9}^{\prime G G} g_{\nu \rho} p_{1 \mu}+\rho_{11}^{\prime G G} g_{\mu \rho} p_{2 \nu}+\rho_{12}^{\prime G G} g_{\nu \rho} p_{2 \mu}+\rho_{14}^{\prime G G} g_{\mu \rho} p_{2 \nu_{\nu}}+\rho_{15}^{\prime G G} g_{\nu \rho} p_{2 \mu}+\ldots
\end{aligned}
$$

After some long and tedious calculations, we find that all of the above coefficients $\rho_{i}^{\prime G G}$ are zero. This is completely the same as for the $\Pi_{\mu \nu}$ case. Therefore, only the perturbative part survives after performing the double Borel transformation on the two variables $p_{1}^{2}$ and $p_{2}^{2}$ at the leading order of the heavy quark mass expansion and QCD running coupling constant expansion for the three-point function $\Pi_{\mu \nu \rho}$.

\section{NUMERICAL RESULTS OF FORM FACTORS IN QCD SUM RULES}

Now we are able to calculate form factors numerically. Firstly, we collect the input parameters used in this work as below [23, 24, 25]

$$
\begin{array}{ll}
m_{c}\left(m_{c}\right)=1.275 \pm 0.015 \mathrm{GeV}, & m_{s}(1 \mathrm{GeV})=142 \mathrm{MeV}, \\
m_{u}(1 \mathrm{GeV})=2.8 \mathrm{MeV}, & m_{d}(1 \mathrm{GeV})=6.8 \mathrm{MeV}, \\
\alpha_{s}(1 \mathrm{GeV})=0.517, & m_{J / \psi}=3.097 \mathrm{GeV}, \\
m_{D^{-}}=1.869 \mathrm{GeV}, & m_{D_{s}^{-}}=1.968 \mathrm{GeV}, \\
m_{D^{*-}}=2.010 \mathrm{GeV}, & m_{D_{s}^{*-}}=2.112 \mathrm{GeV}, \\
f_{J / \psi}=337_{-13}^{+12} \mathrm{MeV}, & f_{D^{-}}=166_{-10}^{+9} \mathrm{MeV}, \\
f_{D_{s}^{-}}=189_{-10}^{+9} \mathrm{MeV}, & f_{D^{*-}}=240_{-10}^{+10} \mathrm{MeV}, \\
f_{D_{s}^{*-}}=262_{-12}^{+9} \mathrm{MeV} . &
\end{array}
$$

All the QCD parameters are adopted at the renormalization scale around $1 \mathrm{GeV}$. It should be pointed out that the mass of charm quark used in this work is determined form the charmonium spectrum in Ref. [23]. 
As for the decay constants of charmed mesons, on the one hand, there is a flood of papers on the theoretical investigation of leptonic decay constants of $D^{+}$and $D_{s}[26,27,28,29,30,31,32,33,34,35,36,37]$; on the other hand, the measurements of decay constants of pseudoscalar $D^{+}$and $D_{s}$ mesons have recently been improved by the CLEO and BaBar collaborations [38, 39]. Moreover, the CLEO collaboration reported their work on the value of ratio $f_{D_{s}^{+}} / f_{D^{+}}$using the measurement of $D_{s}^{+} \rightarrow l^{+} \nu$ channel and obtained $f_{D_{s}^{+}}=274 \pm 13 \pm 7 \mathrm{MeV}$ [41, 42]. However, the decay constants of $D^{*+}$ and $D_{s}^{*}$ mesons have not been directly measured in experiments so far. The only available results on $f_{D^{*+}}$ and $f_{D_{s}^{* 0}}$ from the Lattice QCD calculations [29, 37, 40] determine $f_{D_{s}^{*}}=272 \pm 16_{-20}^{+3} \mathrm{MeV}$ that is smaller than the value of decay constant for $D_{s}^{+}$measured by the CLEO collaboration [41, 42]. To reduce the theoretical uncertainties in the three-point sum rules of the weak transition form factors, due to quarks masses, threshold parameters and Coulomb-like corrections of $J / \psi$ effectively [43], we use the decay constants $f_{\psi}$ and $f_{D_{d, s}^{(*)-}}$ calculated from the two-point QCD sum rules in leading order of $\alpha_{s}$, the same as that in the three-point sum rules. The explicit calculations of the decay constants, in the framework of QCD sum rules, for both $J / \psi$ and $D_{d, s}^{(*)}$ are displayed in Appendix C. Our results indicae that $\frac{f_{D_{s}^{*}}}{f_{p}} \simeq \frac{f_{D_{s}}}{f_{D}}=1.1$, which are in good agreement with that from lattice simulation [40] and experiments [41, 42].

For the threshold parameters $s_{1}^{0}$ and $s_{2}^{0}$, one should determine them by demanding the QCD sum rules results to be relatively stable in allowed regions for $M_{1}^{2}$ and $M_{2}^{2}$, the values of which should be around the mass square of the corresponding first excited states. As for the heavy-light mesons, the standard value of the threshold in the $X$ channel would be $s_{X}^{0}=\left(m_{X}+\Delta_{X}\right)^{2}$, where $\Delta_{X}$ is about 0.6 $\mathrm{GeV}[44,45,46,47,48]$, and we simply take it as $(0.6 \pm 0.1) \mathrm{GeV}$ for the error estimate in the numerical analysis. When it comes to the heavy quarkonium, following the method in Ref. [45, 46, 48], we select the effective threshold parameter to ensure the appearance of the pleasant platform and also around the mass square of $\psi(2 S)$. In this way, the contributions from both the excited states including $\psi(2 S)$ and the continuum states are contained in the spectral function.

\section{A. The numerical results of the form factors}

\section{Evaluation of the form factors for the $J / \psi \rightarrow D^{-}$}

With all the parameters listed above, we can obtain the numerical values of the form factors. The form factors should not depend on the Borel masses $M_{1}$ and $M_{2}$ in a complete theory. However, as we truncate the operator product expansion up to dimension-5 and keep the perturbative expansion in $\alpha_{s}$ to leading order, an obvious dependence of the form factors on these two Borel parameters would emerge. Therefore, one should look for a region where the results only mildly vary with respect to the Borel 
masses, so that the truncation is reasonable and acceptable.

With a careful analysis, $s_{1}^{0}=13.7 \mathrm{GeV}^{2}$ and $s_{2}^{0}=6.1 \mathrm{GeV}^{2}$ are chosen for the form factor $V$ calculation. We require the contributions from the higher states to be less than $30 \%$ and the value of $V$ does not vary drastically within the selected region for the Borel masses. As commonly understood, the Borel parameters $M_{1}^{2}$ and $M_{2}^{2}$ should not be too large in order to insure that the contributions from the higher excited states and continuum are not too significant. On the other hand, the Borel masses also could not be too small for the sake of validity of OPE in the deep Euclidean region, since the contributions of higher dimension operators pertain to the higher orders in $\frac{1}{M_{i}}(i=1,2)$. Different from that adopted in previous literature [11, 15] where the ratio of $M_{1}$ and $M_{2}$ was fixed, in the calculation of form factors, we let $M_{1}$ and $M_{2}$ vary independently as suggested by the authors of Ref. [18, 49]. In this way, we indeed find a Borel platform $M_{1}^{2} \in[6.0,10.0] \mathrm{GeV}^{2}, M_{2}^{2} \in[1.0,2.0] \mathrm{GeV}^{2}$, plotted in Fig. 2, which satisfy the conditions discussed above. One can directly read from this figure that $V\left(q^{2}=0\right)$ is $0.48_{-0.05}^{+0.07}$, whose uncertainties originate from the variation of the Borel parameters.

Following the same procedure, we also obtain numerical results for the other three form factors $A_{0}, A_{1}$ and $A_{2}$ within the chosen Borel window as shown in Fig. 2. The numerical results of form factors $V, A_{0}, A_{1}$ and $A_{2}$ at zero momentum transfer are then

$$
V(0)=0.81_{-0.08}^{+0.12}, \quad A_{0}(0)=0.27_{-0.03}^{+0.02}, \quad A_{1}(0)=0.27_{-0.02}^{+0.03}, \quad A_{2}(0)=0.34_{-0.07}^{+0.07}
$$

It needs to be emphasized that the form factors $A_{1}\left(q^{2}\right), A_{2}\left(q^{2}\right)$ and $A_{0}\left(q^{2}\right)$ should satisfy the relation $\left(m_{\psi}+m_{D^{-}}\right) A_{1}(0)+\left(m_{\psi}-m_{D^{-}}\right) A_{2}(0)=2 m_{\psi} A_{0}(0)$ to ensure disappearance of the divergence at the pole $q^{2}=0$. The theoretical uncertainties in the form factors (44) originate from the Borel masses $M_{1}^{2}$ and $M_{2}^{2}$. They are at the level of $15 \%$, which implies stable results from the QCD sum rules approach.

Indeed there are some extra errors originating from the values of $s_{1}^{0}$ and $s_{2}^{0}$ which correspond to the threshold of the higher excited resonances and continuum states for the $J / \psi$ and $D$ channels respectively. In the QCD sum rules approach, the values of threshold parameter is usually in the vicinity of mass square of the first physical excited state, therefore, we do not investigate the dependence of form factors on the threshold parameter in this work as that in Ref. [18, 19], where a larger threshold value of charmonium is adopted. This uncertainty would cause errors in the resultant form factors. Besides, the fluctuations of the charm quark mass can also result in the uncertainties of the form factors, which is evaluated to be at the level of $6-8 \%$. Moreover, the input parameters such as the decay constants of $D$ meson and $J / \psi$ can also bring on additional uncertainties. Combing the errors from various parameters discussed above, the uncertainties on the form factors can be estimated within 20 to 30\%, expected by the general understanding of the theoretical framework. 

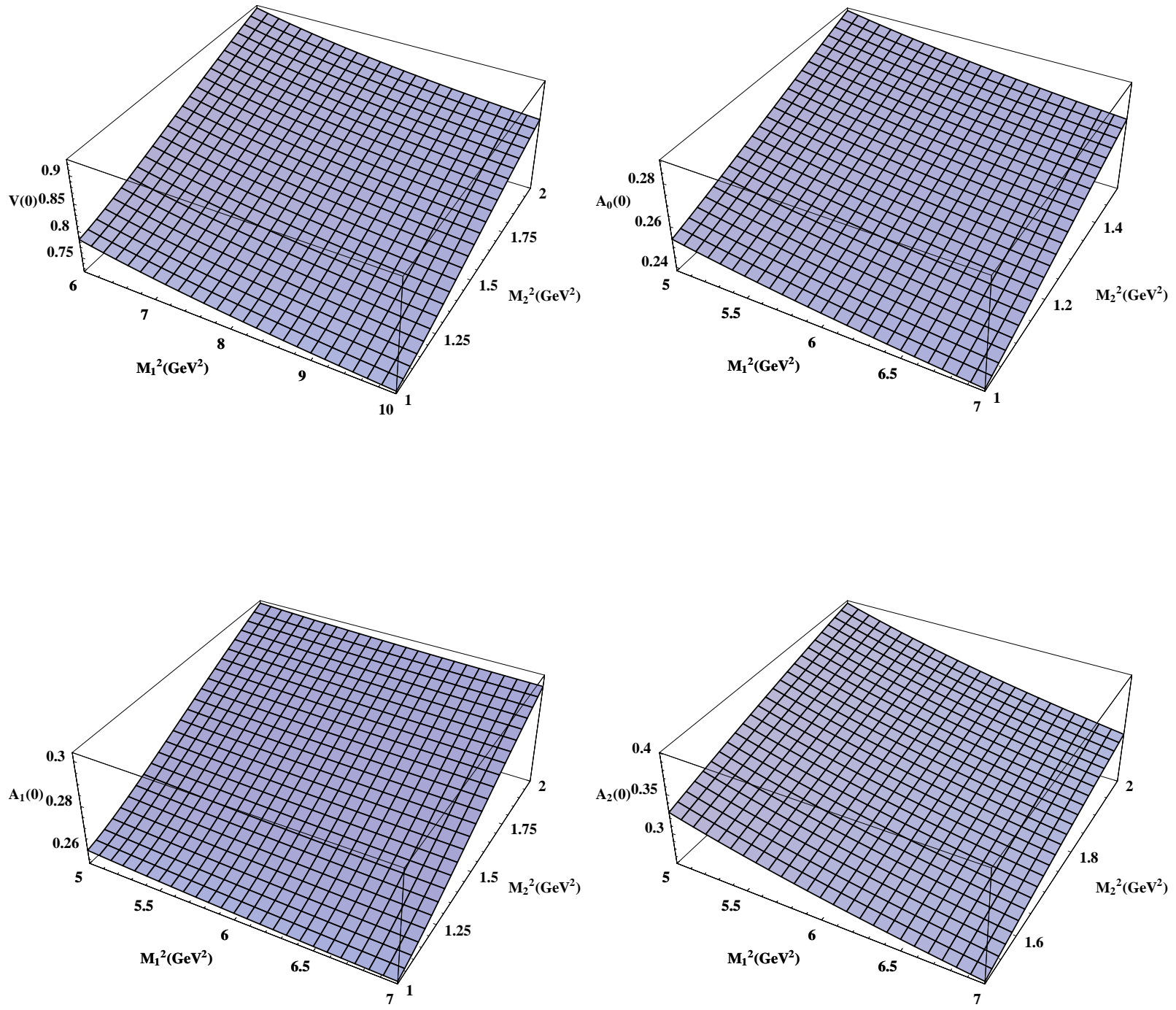

FIG. 2: Dependence of form factors $V, A_{0}, A_{1}$ and $A_{2}$ at $q^{2}=0$ responsible for the decay of $J / \psi \rightarrow D^{-}$on the Borel masses.

Next, we can further investigate the $q^{2}$ dependence of the form factors $V, A_{0}, A_{1}$ and $A_{2}$. The physical region of $q^{2}$ for $J / \psi \rightarrow D^{-} l^{+} \nu_{l}$ is $0 \leq q^{2} \leq\left(m_{J / \psi}-m_{D^{-}}\right)^{2} \simeq 1.5 \mathrm{GeV}^{2}$. However, with the QCD sum rules, we could not obtain the form factors in the whole physical region, since the additional singularities - so called "non-Landau-type" singularities emerge, which had been extensively discussed in Ref.[11]. To avoid this kind of singularity, we restrict our calculations in the range of $q^{2} \in[0,0.47] \mathrm{GeV}^{2}$. We show the $q^{2}$ dependence of the form factors $V, A_{0}, A_{1}$ and $A_{2}$ in Fig. 3 , 


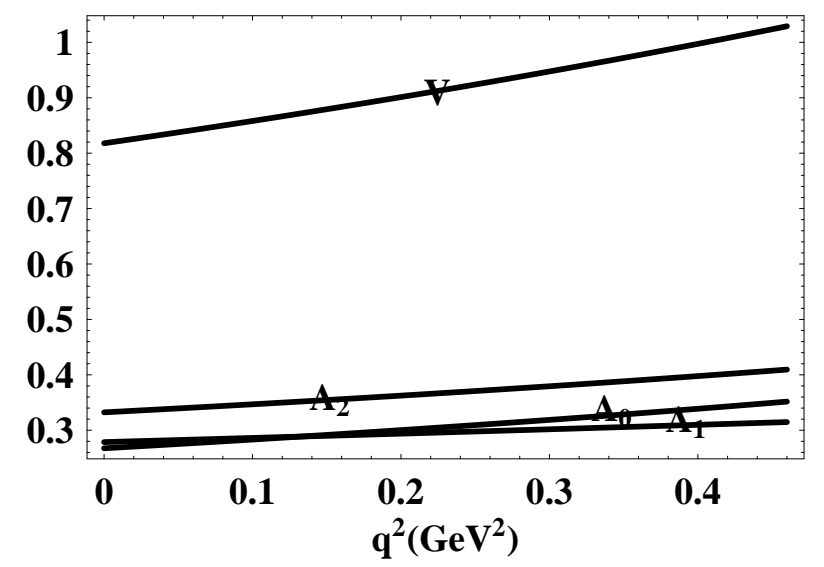

FIG. 3: $q^{2}$ dependence of form factors $V, A_{0}, A_{1}$ and $A_{2}$ for $J / \psi \rightarrow D^{-}$within the kinematical region without non-Landau-type singularities.

In addition, for the convenience of applications to phenomenology, one can parameterize the above form factors in a three-parameter form [50]:

$$
F_{i}\left(q^{2}\right)=\frac{F_{i}(0)}{1-a_{i} q^{2} / m_{D^{-}}^{2}+b_{i} q^{4} / m_{D^{-}}^{4}},
$$

where $F_{i}$ denotes the form factors $V, A_{0}, A_{1}$ and $A_{2}$, and $a_{i}$ and $b_{i}$ are the parameters to be fixed. Using the QCD sum rules $F_{i}\left(q^{2}\right)$ with $q^{2}$ restricted within a certain kinematic region, we can fix the parameters $a_{i}, b_{i}$ in the expression. This double-pole expression for form factors can be generalized to the whole kinematic region. Finally, our results for the parameters $a_{i}, b_{i}$ are given as

$$
\begin{array}{cccc}
a_{V}=1.65_{-0.03}^{+0.20}, & b_{V}=0.76_{-0.09}^{+0.44}, & a_{A_{0}}=1.97_{-0.03}^{+0.15}, & b_{A_{0}}=1.19_{-0.05}^{+0.31}, \\
a_{A_{1}}=0.93_{-0.12}^{+0.27}, & b_{A_{1}}=0.46_{-0.01}^{+0.29}, & a_{A_{2}}=1.47_{-0.16}^{+0.14}, & b_{A_{2}}=0.32_{-0.21}^{+0.19} .
\end{array}
$$

For the other form factors which are discussed in the following subsections, we will adopt the same procedure to obtain the form factors in the whole kinematic region.

\section{2. $J / \psi \rightarrow D_{s}^{-}$form factors}

Now, we move on to the computations of the form factors for the transition $J / \psi \rightarrow D_{s}^{-}$, which is quite similar to that for $J / \psi \rightarrow D^{-}$, only with $d$ quark in $D^{-}$being replaced by $s$. It is also noted that the threshold parameter $s_{2}^{0}=6.6 \mathrm{GeV}^{2}$ for the $D_{s}$ channel and the Borel window are shifted slightly compared with that of $J / \psi \rightarrow D^{-}$. Since the figures are very similar to the case for $J / \psi \rightarrow D^{-}$, we just omit them. The obtained form factors for $J / \psi \rightarrow D_{s}^{-}$at $q^{2}=0$ are

$$
V(0)=1.07_{-0.02}^{+0.05}, \quad A_{0}(0)=0.37_{-0.02}^{+0.02}, \quad A_{1}(0)=0.38_{-0.01}^{+0.02}, \quad A_{2}(0)=0.35_{-0.07}^{+0.08} .
$$




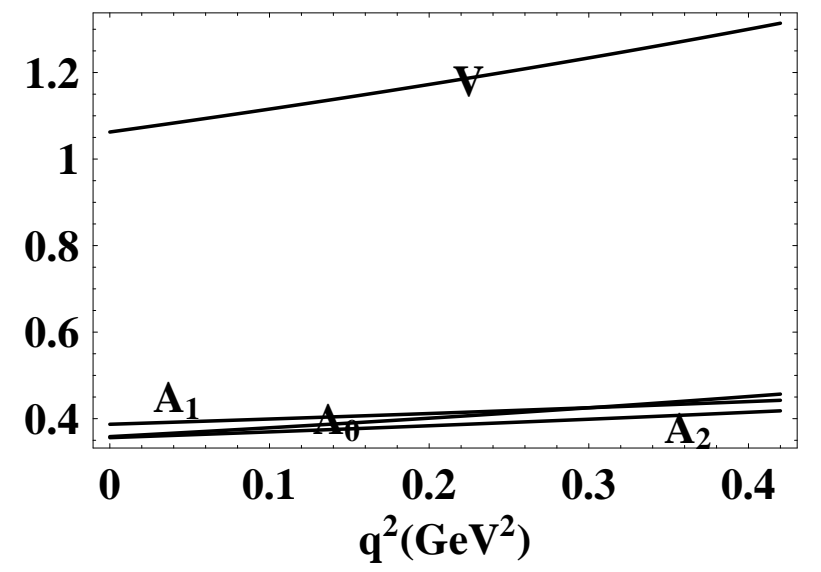

FIG. 4: $q^{2}$ dependence of form factors $V, A_{0}, A_{1}$ and $A_{2}$ for $J / \psi \rightarrow D_{s}^{-}$within the kinematical region without non-Landau-type singularities.

And the parameters $a_{i}$ and $b_{i}$ defined above for the $q^{2}$ dependence formula (with the replacement of $\left.D \rightarrow D_{s}\right)$ are fixed as

$$
\begin{array}{cccc}
a_{V}=1.86_{-0.03}^{+0.26}, & b_{V}=0.90_{-0.04}^{+0.43}, & a_{A_{0}}=2.12_{-0.04}^{+0.0}, & b_{A_{0}}=1.30_{-0.04}^{+0.0}, \\
a_{A_{1}}=1.18_{-0.01}^{+0.24}, & b_{A_{1}}=0.27_{-0.04}^{+0.29}, & a_{A_{2}}=1.41_{-0.29}^{+0.20}, & b_{A_{2}}=0.38_{-0.01}^{+0.15} .
\end{array}
$$

Again, the relation $\left(m_{\psi}+m_{D_{s}}\right) A_{1}(0)+\left(m_{\psi}-m_{D_{s}}\right) A_{2}(0)=2 m_{\psi} A_{0}(0)$ is well respected, which guarantees that the hadronic matrix element responsible for the $J / \psi \rightarrow D_{s}^{-}$is free of divergence due to the pole at $q^{2}=0$. We show the dependence of the form factors on $q^{2}$ in Fig.4.

\section{3. $J / \psi \rightarrow D^{*-}$ form factors}

The evaluation of the form factors responsible for $J / \psi \rightarrow D^{*-}$ is performed following the standard procedure, with appropriate Borel windows obtained. The threshold value for $D^{*-}$ channel takes $s_{2}^{0}=$ $6.8 \mathrm{GeV}^{2}$ in our numerical analysis. The form factors at zero momentum transfer $q^{2}=0$ are collected below as

$$
\begin{array}{llll}
\tilde{A}_{1}(0)=0.40_{-0.01}^{+0.03}, & \tilde{A}_{2}(0)=0.44_{-0.04}^{+0.10}, & \tilde{A}_{3}(0)=0.86_{-0.01}^{+0.05}, & \tilde{A}_{4}(0)=0.91_{-0.04}^{+0.06}, \\
\tilde{V}_{1}(0)=0.41_{-0.01}^{+0.01}, & \tilde{V}_{2}(0)=0.63_{-0.04}^{+0.01}, & \tilde{V}_{3}(0)=0.22_{-0.01}^{+0.03}, & \tilde{V}_{4}(0)=0.26_{-0.05}^{+0.03}, \\
\tilde{V}_{5}(0)=1.37_{-0.03}^{+0.08}, & \tilde{V}_{6}(0)=0.87_{-0.01}^{+0.05} & &
\end{array}
$$


From the above results, we find that the form factors obtained in the QCD sum rules respect the relations $\tilde{A}_{1}(0)=\tilde{A}_{2}(0)$ and $\tilde{V}_{3}(0)=\tilde{V}_{4}(0)$, which are essential to assure that the hadronic matrix element of $J / \psi \rightarrow D^{*-}$ is free of divergence at $q^{2}=0$.

Different from that discussed for the $J / \psi \rightarrow D_{d, s}^{-}$case, not all the form factors which appear in the hadronic matrix element for $J / \psi \rightarrow D^{*-}$, are suitably parameterized in the form of eq.(45) with the thee-parameter approximation. To be more specific, the $q^{2}$ dependence of the form factors $\tilde{A}_{1}\left(q^{2}\right)$ and $\tilde{A}_{2}\left(q^{2}\right)$ are written in the following form [51, 52$]$

$$
F_{i}\left(q^{2}\right)=\frac{F_{i}(0)}{\left(1-a_{i} q^{2} / m_{D^{*-}}^{2}\right)^{2}}
$$

where $F_{i}$ represents $\tilde{A}_{1}$ and $\tilde{A}_{2}$; while the other eight form factors are written in the three-parameter form,

$$
G_{i}\left(q^{2}\right)=\frac{G_{i}(0)}{1-a_{i} q^{2} / m_{D^{*-}}^{2}+b_{i} q^{4} / m_{D^{*-}}^{4}},
$$

where $G_{i}$ can be $\tilde{A}_{3}, \tilde{A}_{4}$ and $\tilde{V}_{i}(i=1-6)$. We then extend the form factors to the whole physical region $0 \leq q^{2} \leq\left(m_{J / \psi}-m_{D^{*-}}\right)^{2} \simeq 1.2 \mathrm{GeV}^{2}$, by fitting the parameters as

$$
\begin{array}{llll}
a_{\tilde{A}_{1}}=1.77_{-0.01}^{+0.04}, & a_{\tilde{A}_{2}}=1.95_{-0.25}^{+0.17}, & a_{\tilde{A}_{3}}=2.93_{-0.08}^{+0.18}, & b_{\tilde{A}_{3}}=2.47_{-0.27}^{+0.54}, \\
a_{\tilde{A}_{4}}=2.78_{-0.03}^{+0.05}, & b_{\tilde{A}_{4}}=1.78_{-0.14}^{+0.27}, & a_{\tilde{V}_{1}}=1.96_{-0.03}^{+0.03}, & b_{\tilde{V}_{1}}=0.98_{-0.06}^{+0.07}, \\
a_{\tilde{V}_{2}}=2.11_{-0.04}^{+0.04}, & b_{\tilde{V}_{2}}=0.21_{-0.02}^{+0.05}, & a_{\tilde{V}_{3}}=1.92_{-0.03}^{+0.0}, & b_{\tilde{V}_{3}}=1.87_{-0.12}^{+0.12}, \\
a_{\tilde{V}_{4}}=2.96_{-0.23}^{+0.34}, & b_{\tilde{V}_{4}}=1.97_{-0.34}^{+1.03}, & a_{\tilde{V}_{5}}=1.92_{-0.12}^{+0.0}, & b_{\tilde{V}_{5}}=1.03_{-0.21}^{+0.0}, \\
a_{\tilde{V}_{6}}=2.00_{-0.02}^{+0.27}, & b_{\tilde{V}_{6}}=1.08_{-0.03}^{+0.53} . &
\end{array}
$$

\section{The form factors for $J / \psi \rightarrow D_{s}^{*-}$}

The computation on the amplitude of $J / \psi \rightarrow D_{s}^{*-}$ is almost the same as that for $J / \psi \rightarrow D^{*-}$, only $d$ quark in $D^{*-}$ being replaced by $s$ quark, with the difference resulting in a different Borel platform. Besides, the threshold parameter for the $D_{s}^{*-}$ channel is set as $s_{2}^{0}=7.4 \mathrm{GeV}^{2}$ in the calculations. The $q^{2}$ dependence of the form factors falling into the region of $q^{2} \in[0,0.37] \mathrm{GeV}^{2}$ is plotted in Fig. 6. As mentioned before, the form factors $\tilde{A}_{1}$ and $\tilde{A}_{2}$ can be parameterized in the form of Eq. (50), while the other form factors can be fit in the usual three-parameter form in Eq.(51). The parameters $a_{i}$ and $b_{i}$ can be determined by reproducing the numbers obtained from the QCD sum rules for the kinematic region $q^{2} \in[0,0.37] \mathrm{GeV}^{2}$ and then we generalize the results to the whole physical region $q^{2} \in[0,0.97] \mathrm{GeV}^{2}$. The values of these parameters together with the form factors at $q^{2}=0$ are collected for convenience as, 

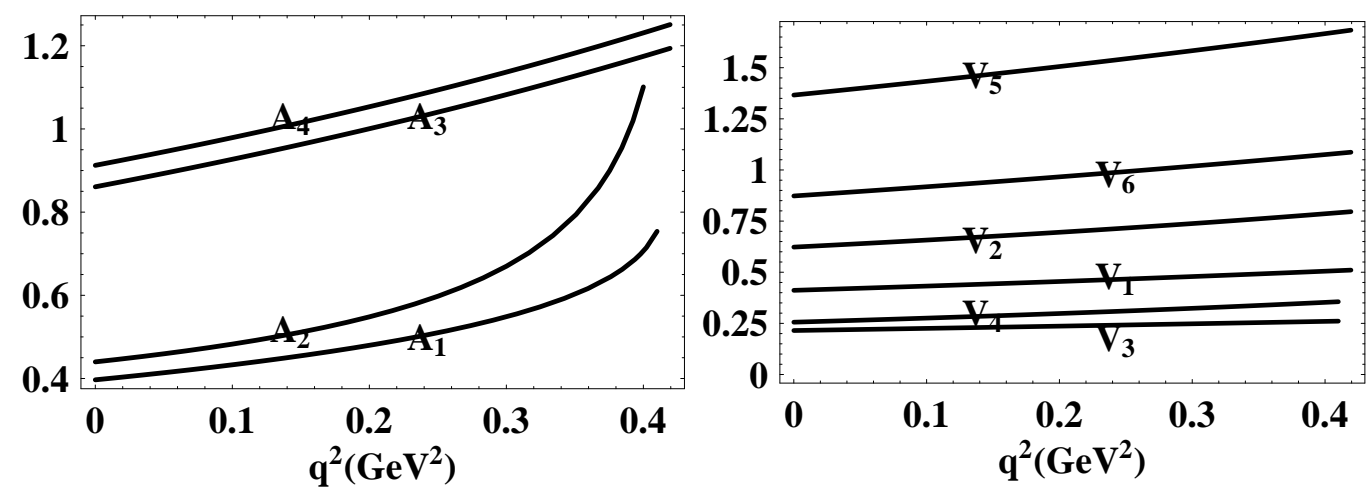

FIG. 5: $q^{2}$ dependence of form factors $\tilde{A}_{1}, \tilde{A}_{2}, \tilde{A}_{3}, \tilde{A}_{4}, \tilde{V}_{1}, \tilde{V}_{2}, \tilde{V}_{3}, \tilde{V}_{4}, \tilde{V}_{5}$ and $\tilde{V}_{6}$ for $J / \psi \rightarrow D^{*-}$ within the kinematical region without non-Landau-type singularities.

$$
\begin{array}{llll}
a_{\tilde{A}_{1}}=1.92_{-0.05}^{+0.0}, & a_{\tilde{A}_{2}}=1.85_{-0.21}^{+0.01}, & a_{\tilde{A}_{3}}=3.07_{-0.01}^{+0.12}, & b_{\tilde{A}_{3}}=1.98_{-0.16}^{+0.80}, \\
a_{\tilde{A}_{4}}=3.08_{-0.02}^{+0.06}, & b_{\tilde{A}_{4}}=2.08_{-0.26}^{+0.60}, & a_{\tilde{V}_{1}}=2.05_{-0.02}^{+0.13}, & b_{\tilde{V}_{1}}=0.90_{-0.06}^{+0.29}, \\
a_{\tilde{V}_{2}}=2.53_{-0.12}^{+0.06}, & b_{\tilde{V}_{2}}=0.07_{-0.35}^{+0.17}, & a_{\tilde{V}_{3}}=2.04_{-0.12}^{+0.16}, & b_{\tilde{V}_{3}}=2.14_{-0.0}^{+0.07}, \\
a_{\tilde{V}_{4}}=3.32_{-0.33}^{+0.37}, & b_{\tilde{V}_{4}}=1.76_{-0.67}^{+1.58}, & a_{\tilde{V}_{5}}=1.92_{-0.03}^{+0.22}, & b_{\tilde{V}_{5}}=0.81_{-0.05}^{+0.40}, \\
a_{\tilde{V}_{6}}=2.00_{-0.04}^{+0.26}, & b_{\tilde{V}_{6}}=0.81_{-0.15}^{+0.55} & &
\end{array}
$$

and

$$
\begin{array}{llll}
\tilde{A}_{1}(0)=0.53_{-0.01}^{+0.03}, & \tilde{A}_{2}(0)=0.53_{-0.01}^{+0.05}, & \tilde{A}_{3}(0)=0.91_{-0.01}^{+0.05}, & \tilde{A}_{4}(0)=0.91_{-0.01}^{+0.06}, \\
\tilde{V}_{1}(0)=0.54_{-0.01}^{+0.01}, & \tilde{V}_{2}(0)=0.69_{-0.06}^{+0.05}, & \tilde{V}_{3}(0)=0.24_{-0.01}^{+0.03}, & \tilde{V}_{4}(0)=0.26_{-0.03}^{+0.03}, \\
\tilde{V}_{5}(0)=1.69_{-0.03}^{+0.10}, & \tilde{V}_{6}(0)=1.14_{-0.01}^{+0.08} & &
\end{array}
$$

In the same way, the relations $\tilde{A}_{1}(0)=\tilde{A}_{2}(0)$ and $\tilde{V}_{3}(0)=\tilde{V}_{4}(0)$ are well satisfied.

\section{DECAY RATES FOR SEMI-LEPTONIC WEAK DECAYS OF $J / \psi$}

With the form factors derived above, we can perform calculations on partial widths of the semi-leptonic decays of $J / \psi$. The relevant CKM parameters are directly taken from the particle data book [24]:

$$
\left|V_{c d}\right|=0.2271, \quad\left|V_{c s}\right|=0.973 .
$$



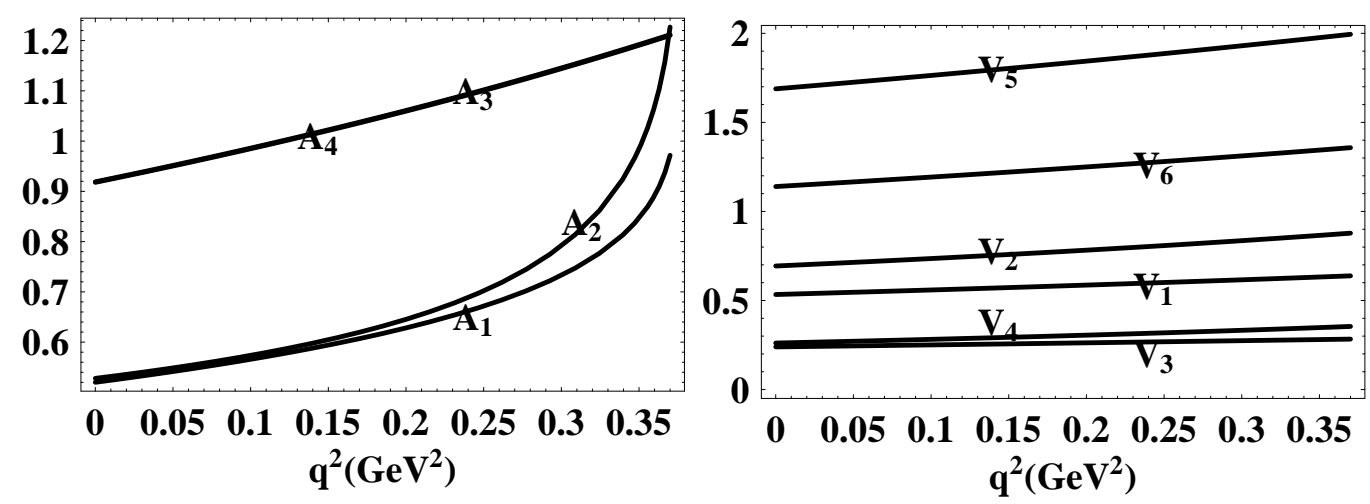

FIG. 6: $q^{2}$ dependence of form factors $\tilde{A}_{1}, \tilde{A}_{2}, \tilde{A}_{3}, \tilde{A}_{4}, \tilde{V}_{1}, \tilde{V}_{2}, \tilde{V}_{3}, \tilde{V}_{4}, \tilde{V}_{5}$ and $\tilde{V}_{6}$ for $J / \psi \rightarrow D_{s}^{*-}$ within the kinematical region without non-Landau-type singularities.

For the semi-leptonic decays $J / \psi \rightarrow D_{(d, s)}^{(*)-} l^{+} \nu_{l}(l=e, \mu)$, the differential partial decay rate is written as

$$
\frac{d \Gamma_{\psi \rightarrow D_{(d, s)}^{(*)} l+\nu_{l}}}{d q^{2}}=\frac{1}{3} \frac{1}{(2 \pi)^{3}} \frac{1}{32 m_{\psi}^{3}} \int_{u_{\min }}^{u_{\max }}\left|\widetilde{M}_{\psi \rightarrow D_{(d, s)}^{(*)-} l^{+} \nu_{l}}\right|^{2} d u
$$

where $u=\left(p_{l^{+}}+p_{\nu_{l}}\right)^{2} ; p_{l^{+}}$and $p_{\nu_{l}}$ are the momenta of $l^{+}$and $\nu_{l}$ respectively; $|\widetilde{M}|^{2}$ is square of the transition amplitude after integrating over the angle between the $l^{+}$and $D_{(d, s)}^{(*)-}$. The upper and lower bounds for $u$ are given as

$$
u_{\min }=\frac{m_{\psi}^{2}-m_{D_{(d, s)}^{(*)}}^{2}-q^{2}}{2 \sqrt{q^{2}}}, \quad u_{\max }=\frac{q^{2}+m_{l}^{2}}{2 \sqrt{q^{2}}} .
$$

The transition amplitude $\widetilde{M}$ for $J / \psi \rightarrow D_{(d, s)}^{(*)-} l^{+} \nu_{l}$ reads as

$$
\widetilde{M}_{\psi \rightarrow D_{(d, s)}^{(*)-} l^{+} \nu_{l}}=\frac{G_{F}}{\sqrt{2}} V_{c q}^{*}\left\langle D_{(d, s)}^{(*)}\left|\bar{q} \gamma_{\mu}\left(1-\gamma_{5}\right) c\right| J / \psi\right\rangle \bar{\nu}_{l} \gamma^{\mu}\left(1-\gamma_{5}\right) l
$$

Finally we get the branching ratios of the semi-leptonic decays as

$$
\begin{aligned}
& \operatorname{BR}\left(J / \psi \rightarrow D^{-} e^{+} \nu_{e}\right)=7.3_{-2.2}^{+4.3} \times 10^{-12}, \quad \operatorname{BR}\left(J / \psi \rightarrow D^{-} \mu^{+} \nu_{\mu}\right)=7.1_{-2.2}^{+4.2} \times 10^{-12}, \\
& \operatorname{BR}\left(J / \psi \rightarrow D_{s}^{-} e^{+} \nu_{e}\right)=1.8_{-0.5}^{+0.7} \times 10^{-10}, \quad \operatorname{BR}\left(J / \psi \rightarrow D_{s}^{-} \mu^{+} \nu_{\mu}\right)=1.7_{-0.5}^{+0.7} \times 10^{-10}, \\
& \operatorname{BR}\left(J / \psi \rightarrow D^{*-} e^{+} \nu_{e}\right)=3.7_{-1.1}^{+1.6} \times 10^{-11}, \operatorname{BR}\left(J / \psi \rightarrow D^{*-} \mu^{+} \nu_{\mu}\right)=3.6_{-1.1}^{+1.6} \times 10^{-11}, \\
& \operatorname{BR}\left(J / \psi \rightarrow D_{s}^{*-} e^{+} \nu_{e}\right)=5.6_{-1.6}^{+1.6} \times 10^{-10}, \operatorname{BR}\left(J / \psi \rightarrow D_{s}^{*-} \mu^{+} \nu_{\mu}\right)=5.4_{-1.5}^{+1.6} \times 10^{-10},
\end{aligned}
$$

where we have combined various uncertainties in the form factors discussed in last section to determine the final error tolerance in our theoretical calculations. Our predictions are much below the present 
experimental upper bounds [2]: $\mathrm{BR}\left(J / \psi \rightarrow D_{s}^{-} e^{+} \nu_{e}+\right.$ c.c. $)<4.9 \times 10^{-5}, \mathrm{BR}\left(J / \psi \rightarrow D^{-} e^{+} \nu_{e}+\right.$ c.c. $)<$ $1.2 \times 10^{-5}$.

A few remarks are presented in order. Firstly, the sum of the branching fractions of semi-leptonic decays of $J / \psi$ whose final state includes $D_{s}^{-}, D_{s}^{*-}, e$ and $\mu$ and their charge conjugate channels can reach as large as $3.1 \times 10^{-9}$, which is expected to be marginally observed at BESIII. Secondly, it is worthwhile to point out that the decay rates for the dominant semi-leptonic weak decays of $J / \psi$ obtained in Ref. [1] were about $7 \times 10^{-9}$, which are two times greater than that calculated in this work. This discrepancy can attribute to the heavy quark spin symmetry and the non-recoil approximation used in Ref. [1], also to the different methods used to estimate the non-perturbative form factors ${ }^{1}$. Thirdly, the ratio of $R_{1} \equiv \frac{\operatorname{BR}\left(J / \psi \rightarrow D_{s}^{*-} e^{+} \nu_{e}\right)}{\operatorname{BR}\left(J / \psi \rightarrow D_{s}^{-} e^{+} \nu_{e}\right)} \simeq 3.1$ is about 2 times larger than the value calculated in [1], where the assumption of heavy quark spin symmetry and non-recoil approximation were adopted. Forth, the ratios $R_{2} \equiv \frac{\mathrm{BR}\left(J / \psi \rightarrow D_{s}^{-} e^{+} \nu_{e}\right)}{\mathrm{BR}\left(J / \psi \rightarrow D^{-} e^{+} \nu_{e}\right)}$ and $R_{3} \equiv \frac{\mathrm{BR}\left(J / \psi \rightarrow D_{s}^{*-} e^{+} \nu_{e}\right)}{\mathrm{BR}\left(J / \psi \rightarrow D^{*-} e^{+} \nu_{e}\right)}$ should be equal to $\left|\frac{V_{c s}}{V_{c d}}\right|^{2} \simeq 18.4$ under the $\mathrm{SU}(3)$ flavor symmetry limit. Our numerical calculations show that $R_{2} \simeq 24.7$ and $R_{3} \simeq 15.1$, which implies large effect of SU(3) symmetry breaking.

\section{DISCUSSIONS AND CONCLUSIONS}

The charmonium $J / \psi$ meson can decay via the strong and electromagnetic interactions, thus weak decays of $J / \psi$ should be very rare unless there is new physics beyond the standard model to make a substantial contribution. If such weak decays can be measured by the future experiments with sizable branching ratios, it would be a clear signal for new physics.

To make the new physics signal clearly distinguishable from the standard model, a careful study of weak decays in SM is needed. In this work, we calculated the form factors of weak transitions of $J / \psi \rightarrow D_{(d, s)}^{(*)}$ in terms of the QCD sum rules. With the form factors, we estimate the branching ratios of the semileptonic weak decays of $J / \psi$ and find that the sum of the branching ratios corresponding to the dominant modes is about $3.1 \times 10^{-9}$ which may be marginally measured by BES III. The QCD sum rules approach possesses uncontrollable errors as large as 20 to $30 \%$, was confirmed by our numerical results. Moreover, due to a Coulomb-type correction in heavy quarkonium decay (or $B_{c}$ ), which may manifest as the ladder structure in the loop-triangle (as a part of multi-loop diagrams), the spectral function needs to be multiplied by a finite renormalization factor [6, 18, 19, 54]. This would bring up another kind of uncertainty. It is expected that this kind of correction can give birth to the twice multiplication of the

\footnotetext{
${ }^{1}$ In Ref. [1], the ISGW model [53] was employed to compute the single form factor $\eta_{12}$, while we adopt the QCD sum rules to calculate the form factors in this work.
} 
form factors at the maximal momentum transfer. However, in this work, we calculate both the three-point QCD sum rules of the weak transition form factors and the two-point sum rules for the decay constant of $J / \psi$ to the same order of $\alpha_{s}$. Then, it is expected that most uncertainties due to the Coulomb-like corrections are canceled in our calculations; therefore, the Coulomb-like corrections for the $J / \psi$ channel are not included in our calculations. As for the heavy-light mesons, there are no corrections in the power of inverse velocity for it, since the light quark moves relativistically. Therefore, one should explore the sum rules for both three-point and two-point correlation functions up to next-to-leading order in strong coupling constant so that Coulomb-like corrections to the heavy-light mesons can be canceled effectively. Moreover, an explicit calculation of Coulomb-like corrections to the heavy-light vertex in triangle diagram is still not available now, which can be left for further considerations.

One can trust the numerical results to a certain accuracy, at least the order of magnitude is reliable. With these form factors we may continue to estimate the rates of non-leptonic weak decays of $J / \psi$ as

long as the factorization theorem is proved. That would be the contents of our next work [55].

The branching ratios of semi-leptonic weak decays of $J / \psi$ are very small in SM, even though their strong decay modes are OZI-suppressed. Our numerical results indicate that even with a large database which will be collected by the BES III, such weak decay modes may only be marginally observed. Therefore we lay our expectation on our BES III colleagues and hope them to provide sufficiently large database to make this challengeable field more fruitful.

\section{Acknowledgements}

This work is partly supported by National Science Foundation of China under Grant No.10475085, 10625525 and 10475042. The authors would like to thank T.M. Aliev, P. Colangelo, T. Huang, V.V. Kiselev, N. Paver, M.Z. Yang, F.K. Guo, Y.L. Shen and W. Wang for helpful discussions.

\section{APPENDIX A: THE EXPLICIT FORMS OF WILSON COEFFICIENTS FOR $\Pi_{\mu \nu}$}

In this appendix, we would like to show the explicit expressions of Wilson coefficients appearing in Eq.(11]14) after the Borel transformation. As mentioned before, only perturbative part contributes to the correlation function for $J / \psi$ decays to $D_{d, s}^{-}$at the leading order of heavy quark mass expansion and the $\alpha_{s}$ expansion series. Namely, Eq. (10) can be written as

$$
f_{i}=f_{i}^{p e r t} \mathbf{I}+O\left(\alpha_{s}\right)+O\left(1 / m_{h}\right),
$$


with $h$ being the heavy charm quark here. The $f_{i}^{\text {pert }}$ can be related to $\rho_{i}^{\text {pert }}$ defined in Eq. (34) by

$$
f_{i}^{\text {pert }}=\int_{\left(m_{c}+m_{q}\right)^{2}}^{s_{2}^{0}} d s_{2} \int_{s_{1}^{L}}^{s_{1}^{0}} d s_{1} \frac{\rho_{i}^{\text {pert }}\left(s_{1}, s_{2}, q^{2}\right)}{\left(s_{1}-p_{1}^{2}\right)\left(s_{2}-p_{2}^{2}\right)},
$$

or

$$
\hat{\mathcal{B}} f_{i}^{\text {pert }}=\int_{\left(m_{c}+m_{q}\right)^{2}}^{s_{2}^{0}} d s_{2} \int_{s_{1}^{L}}^{s_{1}^{0}} d s_{1} \frac{1}{M_{1}^{2}} \mathrm{e}^{-s 1 / M_{1}^{2}} \frac{1}{M_{2}^{2}} \mathrm{e}^{-s_{2} / M_{2}^{2}} \rho_{i}{ }^{\text {pert }}\left(s_{1}, s_{2}, q^{2}\right) .
$$

The lowest bound of $s_{1}$, i.e., $s_{1}^{L}$ can be determined by the Eq. (31) as

$$
\begin{aligned}
s_{1}^{L}= & -\frac{1}{2 m_{q}^{2}}\left[m_{c}^{4}-\left(2 m_{q}^{2}+s_{2}+q^{2}\right) m_{c}^{2}+m_{q}^{2}+s_{2} q^{2}-m_{q}^{2}\left(s_{2}+q^{2}\right)\right. \\
& \left.+\sqrt{m_{c}^{4}-2\left(m_{q}^{2}+s_{2}\right) m_{c}^{2}+\left(m_{q}^{2}-s_{2}\right)^{2}} \sqrt{m_{c}^{4}-2\left(m_{q}^{2}+q^{2}\right) m_{c}^{2}+\left(m_{q}^{2}-q^{2}\right)^{2}}\right],
\end{aligned}
$$

according to the Landau equation [56, 57].

The obvious forms of $\rho_{i}^{\text {pert }}(i=0,2,4,5)$ are

$$
\begin{aligned}
\rho_{0}^{\text {pert }}\left(s_{1}, s_{2}, q^{2}\right)= & -\frac{3}{4 \pi^{2} \lambda^{3 / 2}}\left[m_{c} \lambda+\left(m_{c}-m_{q}\right) s_{1}\left(2 m_{c}^{2}-2 m_{q}^{2}-s_{1}+s_{2}+q^{2}\right)\right], \\
\rho_{2}^{\text {pert }}\left(s_{1}, s_{2}, q^{2}\right)= & -\frac{3 s_{1}}{2 \pi^{2} \lambda^{5 / 2}}\left\{( m _ { c } - m _ { q } ) s _ { 1 } \left[6 m_{c}^{4}-6\left(2 m_{q}^{2}+s_{1}-s_{2}\right) m_{c}^{2}+6 m_{q}^{4}+\left(s_{1}-s_{2}\right)^{2}\right.\right. \\
& \left.+6 m_{q}^{2}\left(s_{1}-s_{2}\right)\right]+m_{c}\left[2\left(m_{c}-m_{q}\right)\left(2 m_{c}+m_{q}\right)-s_{1}+s_{2}\right] \lambda \\
& \left.+q^{2}\left[2\left(m_{c}-m_{q}\right) s_{1}\left(3 m_{c}^{2}-3 m_{q}^{2}-s_{1}+2 s_{2}\right)+m_{c} \lambda+\left(m_{c}-m_{q}\right) s_{1} q^{2}\right]\right\}, \\
\rho_{4}^{\text {pert }}\left(s_{1}, s_{2}, q^{2}\right)= & \frac{3}{4 \pi^{2} \lambda^{5 / 2}}\left\{\left[-m_{c} \lambda^{2}+\left(m_{c}-m_{q}\right)\left(2 s_{2} m_{c}^{2}+s_{1}\left(2 m_{q}^{2}+s_{1}-s_{2}\right)\right] \lambda\right.\right. \\
& +2\left(m_{c}-m_{q}\right) s_{1}\left[3\left(s_{1}+s_{2}\right) m_{c}^{4}\right. \\
& -2\left(3\left(s_{1}+s_{2}\right) m_{q}^{2}+\left(s_{1}-s_{2}\right)\left(s_{1}+2 s_{2}\right)\right) m_{c}^{2}+\left(s_{1}-s_{2}\right)^{2} s_{2}+3 m_{q}^{4}\left(s_{1}+s_{2}\right) \\
& \left.+2 m_{q}^{2}\left(s_{1}-s_{2}\right)\left(s_{1}+2 s_{2}\right)\right]+\left(m_{c}-m_{q}\right) q^{2}\left[2 s _ { 1 } \left(s_{2}^{2}+\left(-2 m_{c}^{2}+2 m_{q}^{2}+s_{1}\right) s_{2}\right.\right. \\
& \left.\left.\left.+\left(m_{c}^{2}-m_{q}^{2}\right)\left(-3 m_{c}^{2}+3 m_{q}^{2}+4 s_{1}\right)\right)-\left(2 m_{c}^{2}+s_{1}\right) \lambda-4 s_{1}\left(m_{c}^{2}-m_{q}^{2}+s_{2}\right) q^{2}\right]\right\}, \\
\rho_{5}^{\text {pert }}\left(s_{1}, s_{2}, q^{2}\right)= & \frac{3}{8 \pi^{2} \lambda^{3 / 2}}\left\{\lambda\left(m_{q} s_{1}+m_{c} s_{2}-m_{c} q^{2}\right)-2\left(m_{c}-m_{q}\right)\left[\lambda m_{c}^{2}\right.\right. \\
& \left.\left.+\left(m_{c}^{2}-m_{q}^{2}\right) s_{1}\left(m_{c}^{2}-m_{q}^{2}-s_{1}+s_{2}\right)+s_{1}\left(m_{c}^{2}-m_{q}^{2}+s_{2}\right) q^{2}\right]\right\} .
\end{aligned}
$$

In this appendix, we adopt the notion $\lambda \equiv \lambda\left(s_{1}, s_{2}, q^{2}\right)$ for the convenience of writing.

\section{APPENDIX B: THE EXPRESSIONS OF WILSON COEFFICIENTS FOR $\Pi_{\mu \nu \rho}$}

Similarly, we will display the forms of Wilson coefficients emerged in the Eq.(19,28) after performing the Borel transformation. As been discussed in the text, only perturbative part contributes to the threepoint function,

$$
F_{i}=F_{i}^{p e r t} \mathbf{I}+O\left(\alpha_{s}\right)+O\left(1 / m_{h}\right)
$$


The relationship between $F_{i}^{\text {pert }}$, and $\rho_{i}^{\prime p e r t}$ are given as

$$
F_{i}^{\text {pert }}=\int_{\left(m_{c}+m_{q}\right)^{2}}^{s_{2}^{0}} d s_{2} \int_{s_{1}^{L}}^{s_{1}^{0}} d s_{1} \frac{\rho_{i}^{\prime p e r t}\left(s_{1}, s_{2}, q^{2}\right)}{\left(s_{1}-p_{1}^{2}\right)\left(s_{2}-p_{2}^{2}\right)}
$$

or

$$
\hat{\mathcal{B}} F_{i}^{\text {pert }}=\int_{\left(m_{c}+m_{q}\right)^{2}}^{s_{2}^{0}} d s_{2} \int_{s_{1}^{L}}^{s_{1}^{0}} d s_{1} \frac{1}{M_{1}^{2}} \mathrm{e}^{-s 1 / M_{1}^{2}} \frac{1}{M_{2}^{2}} \mathrm{e}^{-s_{2} / M_{2}^{2}} \rho_{i}^{\prime}{ }^{\text {pert }}\left(s_{1}, s_{2}, q^{2}\right)
$$

where the definition of $s_{1}^{L}$ is given in Eq. (A4). The manifest expressions of $\rho_{i}^{\prime \text { pert }}(i=$ $1,4,5,6,7,9,11,12,14,15)$ are displayed as

$$
\begin{aligned}
& \rho_{1}^{\prime \text { pert }}\left(s_{1}, s_{2}, q^{2}\right)=\frac{3}{4 \pi^{2} \lambda^{5 / 2}}\left\{-4 s_{1}^{2} m_{c}^{4}+\left(8 m_{q}^{2} s_{1}^{2}+\left(s_{1}-s_{2}\right)\left(s_{1}+s_{2}\right)^{2}-\left(s_{1}-3 s_{2}\right) \lambda\right) m_{c}^{2}\right. \\
& +2 m_{q}\left(s_{1}+s_{2}\right) \lambda m_{c}-4 m_{q}^{4} s_{1}^{2}+s_{2}\left(3 s_{1}+s_{2}\right)\left(\lambda-\left(s_{1}-s_{2}\right)^{2}\right)-m_{q}^{2}\left(s_{1}+s_{2}\right)\left(s_{1}^{2}-s_{2}^{2}+\lambda\right) \\
& +q^{2}\left[4 s_{1} m_{c}^{4}+\left(3 s_{2}^{2}+10 s_{1} s_{2}-s_{1}\left(8 m_{q}^{2}+s_{1}\right)+\lambda\right) m_{c}^{2}-2 m_{q} \lambda m_{c}+3 s_{2}\left(s_{1}+s_{2}\right)^{2}\right. \\
& \left.\left.+4 m_{q}^{4} s_{1}-s_{2} \lambda+m_{q}^{2}\left(s_{1}^{2}-10 s_{1} s_{2}-3 s_{2}^{2}+\lambda\right)+\left(m_{c}^{2}-m_{q}^{2}+s_{2}\right) q^{2}\left(-s_{1}-3 s_{2}+q^{2}\right)\right]\right\}, \\
& \rho_{4}^{\prime \text { pert }}\left(s_{1}, s_{2}, q^{2}\right)=\frac{3 s_{1}}{4 \pi^{2} \lambda^{5 / 2}}\left\{-4 s_{2} m_{c}^{4}-2\left(-4 s_{2} m_{q}^{2}+s_{1}^{2}+3 s_{2}^{2}-4 s_{1} s_{2}-\lambda\right) m_{c}^{2}+4 m_{q} m_{c} \lambda-4 m_{q}^{4} s_{2}\right. \\
& -\left(s_{1}+3 s_{2}\right)\left(\left(s_{1}-s_{2}\right)^{2}-\lambda\right)+2 m_{q}^{2}\left(s_{1}^{2}-4 s_{1} s_{2}+3 s_{2}^{2}-\lambda\right)+q^{2}\left(4 m_{c}^{4}+4\left(s_{2}-2 m_{q}^{2}\right) m_{c}^{2}\right. \\
& \left.\left.+4 m_{q}^{4}+3\left(s_{1}+s_{2}\right)^{2}-4 m_{q}^{2} s_{2}-\lambda+q^{2}\left(2 m_{c}^{2}-2 m_{q}^{2}-3 s_{1}-s_{2}+q^{2}\right)\right)\right\}, \\
& \rho_{5}^{\prime \text { pert }}\left(s_{1}, s_{2}, q^{2}\right)=\frac{1}{4 \pi^{2} \lambda^{5 / 2}}\left\{-\left(s_{1}+s_{2}-q^{2}\right)\left[3\left(m_{c}^{2}-m_{q}^{2}+s_{2}\right) q^{4}-6\left(\left(2 s_{1}+s_{2}\right) m_{c}^{2}+s_{2}\left(s_{1}+s_{2}\right)\right.\right.\right. \\
& \left.-m_{q}^{2}\left(2 s_{1}+s_{2}\right)\right) q^{2}-3\left(2 s_{1} m_{c}^{4}-\left(4 s_{1} m_{q}^{2}+3 s_{1}^{2}+s_{2}^{2}-\lambda\right) m_{c}^{2}\right. \\
& \left.\left.+2 m_{q} m_{c} \lambda+2 m_{q}^{4} s_{1}+m_{q}^{2}\left(3 s_{1}^{2}+s_{2}^{2}-\lambda\right)+s_{2}\left(s_{1}^{2}-s_{2}^{2}+\lambda\right)\right)\right]-2 s_{2}\left[3 s _ { 1 } \left(-2\left(m_{c}^{2}-m_{q}^{2}\right)^{2}\right.\right. \\
& \left.\left.\left.+s_{1}^{2}-s_{2}^{2}-4\left(m_{c}^{2}-m_{q}^{2}\right) s_{2}\right)-3\left(2 m_{c}^{2}+s_{1}\right) \lambda+3 s_{1} q^{2}\left(q^{2}-2\left(s_{1}+s_{2}\right)\right)\right]\right\}, \\
& \rho_{6}^{\prime \text { pert }}\left(s_{1}, s_{2}, q^{2}\right)=\frac{3}{4 \pi^{2} \lambda^{5 / 2}}\left\{-2 s_{1}\left(3 s_{1}+s_{2}\right) m_{c}^{4}+2\left[s_{1}\left(2 m_{q}^{2}+s_{1}-s_{2}\right)\left(3 s_{1}+s_{2}\right)-\left(2 s_{1}+s_{2}\right) \lambda\right] m_{c}^{2}\right. \\
& -4 m_{q} s_{1} m_{c} \lambda+s_{1}\left[-2\left(3 s_{1}+s_{2}\right) m_{q}^{4}+2\left(-3 s_{1}^{2}+2 s_{1} s_{2}+s_{2}^{2}+\lambda\right) m_{q}^{2}+\left(s_{1}-s_{2}\right)^{2}\left(s_{1}+s_{2}\right)\right. \\
& \left.-\left(s_{1}+3 s_{2}\right) \lambda\right]+q^{2}\left[2 s_{1} m_{c}^{4}+2\left(\lambda-2 s_{1}\left(m_{q}^{2}+2 s_{1}\right)\right) m_{c}^{2}\right. \\
& \left.\left.+s_{1}\left(2 m_{q}^{4}+8 s_{1} m_{q}^{2}-\left(s_{1}+s_{2}\right)\left(3 s_{1}+5 s_{2}\right)+\lambda\right)+s_{1}\left(2 m_{c}^{2}-2 m_{q}^{2}+3 s_{1}+5 s_{2}-q^{2}\right) q^{2}\right]\right\}, \\
& \rho_{7}^{\prime \text { pert }}\left(s_{1}, s_{2}, q^{2}\right)=-\frac{3}{16 \pi^{2} \lambda^{5 / 2}}\left\{\left(2\left(m_{c}-m_{q}\right)^{2}-2 s_{2}\right) \lambda^{2}-2\left[s_{2}\left(-s_{1}+s_{2}-q^{2}\right)+\left(m_{c}^{2}-m_{q}^{2}\right)\left(s_{1}+s_{2}-q^{2}\right)\right]\right. \\
& \times\left(s_{1}-s_{2}+q^{2}\right) \lambda-8\left[\left(m_{c}^{2}-m_{q}^{2}+s_{2}\right)\left(s_{1}+s_{2}-q^{2}\right)-2 s_{1} s_{2}\right]\left[\left(\left(s_{1}+s_{2}-q^{2}\right)^{2}\right.\right. \\
& \left.\left.\left.-4 s_{1} s_{2}\right) m_{c}^{2}+s_{1}\left(m_{c}^{2}-m_{q}^{2}+s_{2}\right)^{2}+s_{1}^{2} s_{2}-s_{1}\left(m_{c}^{2}-m_{q}^{2}+s_{2}\right)\left(s_{1}+s_{2}-q^{2}\right)\right]\right\} \\
& \rho_{9}^{\prime \text { pert }}\left(s_{1}, s_{2}, q^{2}\right)=-\frac{3}{8 \pi^{2} \lambda^{5 / 2}}\left\{-\left(\left(m_{c}-m_{q}\right)^{2}-s_{2}\right) \lambda^{2}-\left[s_{2}\left(-s_{1}+s_{2}-q^{2}\right)+\left(m_{c}^{2}-m_{q}^{2}\right)\left(s_{1}+s_{2}-q^{2}\right)\right]\right. \\
& \times\left(s_{1}-s_{2}+q^{2}\right) \lambda+2\left[\lambda m_{c}^{2}+\left(m_{c}^{2}-m_{q}^{2}\right) s_{1}\left(m_{c}^{2}-m_{q}^{2}-s_{1}+s_{2}\right)+s_{1}\left(m_{c}^{2}-m_{q}^{2}+s_{2}\right) q^{2}\right] \lambda
\end{aligned}
$$




$$
\begin{aligned}
& -4\left[\left(m_{c}^{2}-m_{q}^{2}+s_{2}\right)\left(s_{1}+s_{2}-q^{2}\right)-2 s_{1} s_{2}\right]\left[\left(\left(s_{1}+s_{2}-q^{2}\right)^{2}-4 s_{1} s_{2}\right) m_{c}^{2}\right. \\
& \left.\left.+s_{1}\left(m_{c}^{2}-m_{q}^{2}+s_{2}\right)^{2}+s_{1}^{2} s_{2}-s_{1}\left(m_{c}^{2}-m_{q}^{2}+s_{2}\right)\left(s_{1}+s_{2}-q^{2}\right)\right]\right\}, \\
& \rho_{11}^{\prime \text { pert }}\left(s_{1}, s_{2}, q^{2}\right)=-\frac{3 s_{1}}{8 \pi^{2} \lambda^{5 / 2}}\left\{-\lambda^{2}-\left(-2 m_{c}^{2}+2 m_{q}^{2}+s_{1}-s_{2}-q^{2}\right)\left[-2\left(m_{c}-m_{q}\right)^{2}-s_{1}+s_{2}+q^{2}\right] \lambda\right. \\
& -4\left[\left(\left(s_{1}+s_{2}-q^{2}\right)^{2}-4 s_{1} s_{2}\right) m_{c}^{2}+s_{1}\left(m_{c}^{2}-m_{q}^{2}+s_{2}\right)^{2}+s_{1}^{2} s_{2}\right. \\
& \left.\left.-s_{1}\left(m_{c}^{2}-m_{q}^{2}+s_{2}\right)\left(s_{1}+s_{2}-q^{2}\right)\right]\left(-2 m_{c}^{2}+2 m_{q}^{2}+s_{1}-s_{2}-q^{2}\right)\right\}, \\
& \rho_{12}^{\prime \text { pert }}\left(s_{1}, s_{2}, q^{2}\right)=-\frac{3}{8 \pi^{2} \lambda^{5 / 2}}\left\{s_{1} \lambda^{2}-s_{1}\left(-2 m_{c}^{2}+2 m_{q}^{2}+s_{1}-s_{2}-q^{2}\right)\left(s_{1}+s_{2}-q^{2}\right) \lambda\right. \\
& +2\left[\lambda m_{c}^{2}+\left(m_{c}^{2}-m_{q}^{2}\right) s_{1}\left(m_{c}^{2}-m_{q}^{2}-s_{1}+s_{2}\right)+s_{1}\left(m_{c}^{2}-m_{q}^{2}+s_{2}\right) q^{2}\right] \lambda \\
& -4 s_{1}\left[\left(\left(s_{1}+s_{2}-q^{2}\right)^{2}-4 s_{1} s_{2}\right) m_{c}^{2}+s_{1}\left(m_{c}^{2}-m_{q}^{2}+s_{2}\right)^{2}+s_{1}^{2} s_{2}\right. \\
& \left.\left.-s_{1}\left(m_{c}^{2}-m_{q}^{2}+s_{2}\right)\left(s_{1}+s_{2}-q^{2}\right)\right]\left(-2 m_{c}^{2}+2 m_{q}^{2}+s_{1}-s_{2}-q^{2}\right)\right\}, \\
& \rho_{14}^{\prime \text { pert }}\left(s_{1}, s_{2}, q^{2}\right)=-\frac{3}{4 \pi^{2} \lambda^{7 / 2}}\left\{-2 m_{c}^{2}\left(s_{1}+s_{2}-q^{2}\right) \lambda^{2}-s_{1}\left[4 s_{1} s_{2}\left(-2 m_{c}^{2}+2 m_{q}^{2}+s_{1}-s_{2}-q^{2}\right)\right.\right. \\
& -\left(s_{2}\left(-s_{1}+s_{2}-q^{2}\right)+\left(m_{c}^{2}-m_{q}^{2}\right)\left(s_{1}+s_{2}-q^{2}\right)\right)\left(s_{1}+s_{2}-q^{2}\right) \\
& \left.+3\left(m_{c}^{2}-m_{q}^{2}+s_{2}\right)\left(s_{1}+s_{2}-q^{2}\right)\left(2 m_{c}^{2}-2 m_{q}^{2}-s_{1}+s_{2}+q^{2}\right)\right] \lambda \\
& +4 s_{1}\left[4 s_{2}\left\{\left(s_{1}+s_{2}-q^{2}\right)^{2}+s_{1} s_{2}\right\} s_{1}^{2}-3\left(m_{c}^{2}-m_{q}^{2}+s_{2}\right)\left\{\left(s_{1}+s_{2}-q^{2}\right)^{2}\right.\right. \\
& \left.+6 s_{1} s_{2}\right\}\left(s_{1}+s_{2}-q^{2}\right) s_{1}+2\left(m_{c}^{2}\left(s_{1}+s_{2}-q^{2}\right)^{4}+\left\{6 s_{1}\left(m_{c}^{2}-m_{q}^{2}+s_{2}\right)^{2}\right.\right. \\
& \left.\left.-2 m_{c}^{2} s_{1} s_{2}\right\}\left(s_{1}+s_{2}-q^{2}\right)^{2}+2 s_{1}^{2} s_{2}\left(3\left(m_{c}^{2}-m_{q}^{2}+s_{2}\right)^{2}-4 m_{c}^{2} s_{2}\right)\right)-2\left(m_{c}^{2}-m_{q}^{2}\right. \\
& \left.\left.\left.+s_{2}\right)\left\{3\left(\left(s_{1}+s_{2}-q^{2}\right)^{2}-4 s_{1} s_{2}\right) m_{c}^{2}+5 s_{1}\left(m_{c}^{2}-m_{q}^{2}+s_{2}\right)^{2}\right\}\left(s_{1}+s_{2}-q^{2}\right)\right]\right\} \text {, } \\
& \rho_{15}^{\prime \text { pert }}\left(s_{1}, s_{2}, q^{2}\right)=-\frac{3}{4 \pi^{2} \lambda^{7 / 2}}\left\{-2 m_{c}^{2}\left(s_{1}+s_{2}-q^{2}\right) \lambda^{2}-s_{1}\left(4 s_{1} s_{2}\left(-2 m_{c}^{2}+2 m_{q}^{2}+s_{1}-s_{2}-q^{2}\right)\right.\right. \\
& -\left[s_{2}\left(-s_{1}+s_{2}-q^{2}\right)+\left(m_{c}^{2}-m_{q}^{2}\right)\left(s_{1}+s_{2}-q^{2}\right)\right]\left(s_{1}+s_{2}-q^{2}\right) \\
& \left.+3\left(m_{c}^{2}-m_{q}^{2}+s_{2}\right)\left(s_{1}+s_{2}-q^{2}\right)\left(2 m_{c}^{2}-2 m_{q}^{2}-s_{1}+s_{2}+q^{2}\right)\right) \lambda \\
& +4\left[-10 s_{2}^{2}\left(s_{1}+s_{2}-q^{2}\right) s_{1}^{3}+12 s_{2}\left(m_{c}^{2}-m_{q}^{2}+s_{2}\right)\left(\left(s_{1}+s_{2}-q^{2}\right)^{2}+s_{1} s_{2}\right) s_{1}^{2}\right. \\
& -3\left(2 s_{2}\left(\left(s_{1}+s_{2}-q^{2}\right)^{2}-4 s_{1} s_{2}\right) m_{c}^{2}+\left(m_{c}^{2}-m_{q}^{2}+s_{2}\right)^{2}\left(\left(s_{1}+s_{2}-q^{2}\right)^{2}+6 s_{1} s_{2}\right)\right)\left(s_{1}\right. \\
& \left.+s_{2}-q^{2}\right) s_{1}+2\left(m_{c}^{2}-m_{q}^{2}+s_{2}\right)\left(m_{c}^{2}\left(s_{1}+s_{2}-q^{2}\right)^{4}+2 s_{1}\left(\left(m_{c}^{2}-m_{q}^{2}+s_{2}\right)^{2}\right.\right. \\
& \left.\left.\left.\left.-m_{c}^{2} s_{2}\right)\left(s_{1}+s_{2}-q^{2}\right)^{2}+2 s_{1}^{2} s_{2}\left(\left(m_{c}^{2}-m_{q}^{2}+s_{2}\right)^{2}-4 m_{c}^{2} s_{2}\right)\right)\right]\right\} \text {. }
\end{aligned}
$$




\section{APPENDIX C: DECAY CONSTANTS OF $J / \psi$ AND $D_{d, s}^{(*)}$ IN TWO-POINT QCD SUM RULES}

In this appendix, we would like to collect the sum rules for the decay constants of $J / \psi$ and $D_{d, s}^{(*)}$ for the completeness of the paper. The decay constant of $J / \psi$ in the two-point QCD sum rules can be written as [45, 58$]$

$$
f_{\psi}^{2} m_{\psi}^{2} e^{-\frac{m_{\psi}^{2}}{M^{2}}}=\int_{4 m_{c}^{2}}^{s_{\psi}^{0}} d s \frac{s}{4 \pi^{2}}\left(1-\frac{4 m_{c}^{2}}{s}\right)^{1 / 2}\left(1+\frac{2 m_{c}^{2}}{s}\right) e^{-\frac{s}{M^{2}}}+\left[-\frac{m_{c}^{2}}{4 M^{2}}+\frac{1}{16}+\frac{1}{48} e^{-\frac{4 m_{c}^{2}}{M^{2}}}\right]\left\langle 0\left|\frac{\alpha_{s}}{\pi} G_{\mu \nu}^{2}\right| 0\right\rangle,
$$

where the nonrelativistic approximation for the gluon condensate has been adopted for the convenience of performing the Borel transformation. It is observed that the gluon condensate has tiny effect on the results of the form factors and hence are neglected in the sum rules of charmed mesons. The non-perturbative condensates used in the evaluation of the sum rues can be grouped as

$$
\begin{array}{ll}
\langle 0|\bar{q} q| 0\rangle=-(1.65 \pm 0.15) \times 10^{-2} \mathrm{GeV}^{3}(q=u, d), & \langle 0|\bar{s} s| 0\rangle=(0.8 \pm 0.1)\langle 0|\bar{q} q| 0\rangle, \\
\left\langle 0\left|\frac{\alpha_{s}}{\pi} G_{\mu \nu}^{2}\right| 0\right\rangle=0.005 \pm 0.004 \mathrm{GeV}^{2}, & \left\langle 0\left|\bar{q}_{i} \sigma \cdot G q_{i}\right| 0\right\rangle=m_{0}^{2}\left\langle 0\left|\bar{q}_{i} q_{i}\right| 0\right\rangle,
\end{array}
$$

where $m_{0}^{2}=(0.8 \pm 0.2) \mathrm{GeV}^{2}$ and the subscript " $i$ " denotes the flavor of quarks. Based on the twopoint sum rules of $J / \psi$ and the parameters showed above, we can derive the decay constant of $J / \psi$ as $337_{-13}^{+12} \mathrm{MeV}$, where we have combined the uncertainties from the variations of the Borel masses and threshold value for $J / \psi$ channel.

The sum rules for the decay constant of $D_{q}$ can be given by [도, $\left.\underline{59}, \underline{60}, \underline{61}\right]$

$$
\begin{aligned}
\frac{m_{D_{q}}^{4}}{\left(m_{c}+m_{q}\right)^{2}} f_{D_{q}}^{2} e^{-\frac{m_{D_{q}}^{2}}{M^{2}}}= & \frac{3}{8 \pi^{2}} \int_{\left(m_{c}+m_{q}\right)^{2}}^{s_{D_{q}}^{0}} d s\left[1-\frac{\left(m_{c}-m_{q}\right)^{2}}{s}\right] \lambda^{1 / 2}\left(s, m_{c}^{2}, m_{q}^{2}\right) e^{-\frac{s}{M^{2}}} \\
& +\left(-m_{c}+\frac{m_{q}}{2}+\frac{m_{q} m_{c}^{2}}{2 M^{2}}\right) e^{-\frac{m_{c}^{2}}{M^{2}}}\langle 0|\bar{q} q| 0\rangle-\frac{m_{c}}{2 M^{2}}\left(1-\frac{m_{c}^{2}}{2 M^{2}}\right) e^{-\frac{m_{c}^{2}}{M^{2}}}\langle 0|\bar{q} \sigma \cdot G q| 0\rangle,
\end{aligned}
$$

from which we can arrive at the decay constants of pseudoscalar charmed mesons as $f_{D_{d}}=166_{-10}^{+9} \mathrm{MeV}$ and $f_{D_{s}}=189_{-10}^{+9} \mathrm{MeV}$.

The decay constants of vector charmed mesons $f_{D_{q}^{*}}$ in the framework of QCD sum rules can be calculated as [58, 60, 62]

$$
\begin{aligned}
f_{D_{q}^{*}}^{2} m_{D_{q}^{*}}^{2} e^{-\frac{m_{D_{q}^{*}}^{2}}{M^{2}}}= & \frac{1}{8 \pi^{2}} \int_{\left(m_{c}+m_{q}\right)^{2}}^{s_{D_{q}^{*}}^{0}} d s \lambda^{1 / 2}\left(s, m_{c}^{2}, m_{q}^{2}\right)\left[2-\frac{m_{c}^{2}+m_{q}^{2}-6 m_{c} m_{q}}{s}-\frac{\left(m_{c}^{2}-m_{q}^{2}\right)^{2}}{s^{2}}\right] e^{-\frac{s}{M^{2}}} \\
& +\left\{\left[-\left(m_{c}+\frac{8}{3} m_{q}\right)+\frac{1}{2} \frac{m_{q} m_{c}^{2}}{M^{2}}\right] e^{-\frac{m_{c}^{2}}{M^{2}}}+\frac{2 m_{q}\left(4 M^{2}-m_{c}^{2}\right)}{3 M^{2}}\right\}\langle 0|\bar{q} q| 0\rangle \\
& +\frac{m_{c}^{3}}{4 M^{4}} e^{-\frac{m_{c}^{2}}{M^{2}}}\langle 0|\bar{q} \sigma \cdot G q| 0\rangle,
\end{aligned}
$$


from which we can achieve the decay constants of vector charmed mesons as $f_{D_{d}^{*}}=240_{-10}^{+10} \mathrm{MeV}$ and $f_{D_{s}^{*}}=262_{-12}^{+9} \mathrm{MeV}$.

[1] M.A. Sanchis-Lonzano, Z. Phys. C 62, 271 (1994).

[2] M. Ablikim et al.[BES Collaboration], Phys. Lett. B 639, 418 (2006) [arXiv: hep-ex/0604005.

[3] F.A. Harris, arXiv: hep-ex/0606059.

[4] M. Wirbel, B. Stech, M. Bauer, Z. Phys. C 29, 637 (1985).

[5] M. Terent'ev, Sov. J. Nucl. Phys. 24, 106 (1976); V. Berestetsky and M. Terent'ev, ibid. 24, 547 (1976); 25, 347 (1977); P. Chung, F. Coester, and W. Polyzou, Phys. Lett. B 205, 545 (1988); W. Jaus, Phys. Rev. D 41, 3394 (1990); 44, 2851 (1991); 60, 054026 (1999). C. Ji, P. Chung and S. Cotanch, Phys. Rev. D 45, 4214 (1992); H.Y. Cheng, C.Y. Cheung and C.W. Hwang, Phys. Rev. D 55, 1559 (1997) [arXiv: hep-ph/9607332; H.Y. Cheng, C.K. Chua and C.W. Hwang, Phys. Rev. D 69, 074025 (2004) [arXiv: hep-ph/0310359; C.W. Hwang and Z.T. Wei, J. Phys. G 34, 687 (2007) [arXiv: hep-ph/0609036]; C.D. Lü, W. Wang and Z.T. Wei, Phys. Rev. D 76, 014013 (2007) [arXiv: hep-ph/0701265.

[6] M. A. Shifman, A. I. Vainshtein and V. I. Zakharov, Nucl. Phys. B 147, 385 (1979).

[7] V. A. Novikov, M. A. Shifman, A. I. Vainshtein and V. I. Zakharov, Nucl.Phys. B 191, 301 (1981).

[8] Y.Y. Keum, H.N. Li, and A.I. Sanda, Phys. Lett. B 504, 6 (2001) [arXiv: hep-ph/0004004; C.D. Lü, K. Ukai, and M.Z. Yang, Phys. Rev. D 63, 074009 (2001) [arXiv: hep-ph/0004213]; T. Kurimoto, H.n. Li, A.I. Sanda, Phys. Rev. D 65, 014007 (2002) [arXiv: hep-ph/0105003]; Phys. Rev. D 67, 054028 (2003) [arXiv: hep-ph/0210289; Z.T. Wei, M.Z. Yang, Nucl. Phys. B 642, 263 (2002) [arXiv: hep-ph/0202018; C.D. Lu and M.Z. Yang, Eur. Phys. J. C 28, 515 (2003) [arXiv: hep-ph/0212373].

[9] B.L. Ioffe and A.V. Smilga, Phys. Lett. B114, 353 (1982); Nucl. Phys. B 216, 373 (1983).

[10] V.A. Nesterenko and A.V. Radyushkin, Phys. Lett. B115, 410 (1982).

[11] P. Ball, V.M. Braun and H.G. Dosch, Phys. Rev. D 44, 3567 (1991).

[12] For a review of QCD sum rules applicaions to weak decays of heavy mesons, see A. Khodjamirian and R. Ruckl, Adv. Ser. Direct. High Energy Phys. 15, 345 (1998) [arXiv: hep-ph/9801443].

[13] V.L. Eletsky, B.L. Ioffe and Ya.I. Kogan, Phys. Lett. B 122, 423 (1983).

[14] V.L. Chernyak and A.R. Zhitnitsky, Phys. Rep. 112, 173 (1984);V.L. Chernyak, A.A. Ogloblin and I.R. Zhitnitsky, Z. Phys. C 42, 569 (1989); P. Ball, V. M. Braun, Y. Koike and K. Tanaka, Nucl. Phys. B 529, 323 (1998) [arXiv: hep-ph/9802299]; P. Ball, V. M. Braun, Nucl. Phys. B 529, 323 (1998) [arXiv: hep-ph/9810475]; P. Ball, JHEP 9901, 010 (1999) [arXiv: hep-ph/9812375]; H.Y. Cheng, C.K. Chua and K.C. Yang, Phys. Rev. D 73, 014017 (2006) [arXiv: hep-ph/0508104]; C.D. Lü, Y.M. Wang and H. Zou, Phys. Rev. D 75, 056001 (2007) [arXiv: hep-ph/0612210.

[15] P. Ball, Phys. Rev. D 48, 3190 (1993) [arXiv: hep-ph/9305267.

[16] M. Wirbel, B. Stech and M. Bauer, Z. Phys. C 29, 637 (1985). 
[17] Talk given by A. Kagan at 4th International Workshop on the CKM Unitarity Triangle (CKM 2006), Nagoya, Japan, 12-16 Dec 2006.

[18] V.V. Kiselev, A.K. Likhoded and A.I. Onishchenko, Nucl. Phys. B 569, 473 (2000) [arXiv: hep-ph/9905359.

[19] V.V. Kiselev, A.E. Kovalsky and A.K. Likhoded, Nucl. Phys. B 585, 353 (2000) [arXiv: hep-ph/0002127].

[20] T.M. Aliev and M. Savci, Eur. Phys. J. C 47, 413 (2006) [arXiv: hep-ph/0601267].

[21] D.S. Du, J.W. Li and M.Z. Yang, Eur. Phys. J. C 37, 173 (2004) [arXiv: hep-ph/0308259.

[22] M.Z. Yang, Phys. Rev. D 73, 034027 (2006) [arXiv: hep-ph/0509103; D 73, 079901 (2006) (E).

[23] B.L. Ioffe, Prog. Part. Nucl. Phys. 56, 232 (2006) [arXiv: hep-ph/0502148].

[24] W.M. Yao et al., J. Phys. G 33, 1 (2006).

[25] M.A. Ivanov, J.G. Körner and P. Santorelli, Phys. Rev. D 73, 054024 (2006) [arXiv: hep-ph/0602050.

[26] A. Ali Khan, V. Braun, T. Burch, M. Gockeler, G. Lacagnina, A. Schafer, G. Schierholz, [arXiv: hep-lat/0701015.

[27] H.-M. Choi, Phys. Rev. D 75, 073016 (2007) [arXiv: hep-ph/0701263].

[28] D. Ebert, R.N. Faustov and V.O. Galkin, Phys. Lett. B 635, 93 (2006) [arXiv: hep-ph/0602110].

[29] C. Aubin et al., Phys. Rev. Lett. 95, 122002 (2005) [arXiv: hep-lat/0506030.

[30] T. W. Chiu, T.H. Hsieh, J.Y. Lee, P.H. Liu and H.J. Chang, Phys. Lett. B 624, 31 (2005) [arXiv: hep-ph/0506266.

[31] L. Lellouch and C.-J. Lin (UKQCD Collaboration), Phys. Rev. D 64, 094501 (2001) [arXiv: hep-ph/0011086].

[32] J. Bordes, J. Peñarrocha, and K. Schilcher, JHEP 0511, 014 (2005) [arXiv: hep-ph/0507241.

[33] S. Narison, arXiv: hep-ph/0202200.

[34] A. Juttner and J. Rolf, Invited talk at 2nd Workshop on the CKM Unitarity Triangle, Durham, England, 5-9 April, 2003 [arXiv: hep-ph/0306299].

[35] A. Khodjamirian, Invited talk at 2nd Workshop on the CKM Unitarity Triangle, Durham, England, 5-9 April, 2003 [arXiv: hep-ph/0306253.

[36] D. Becirevic, Nucl. Phys. Proc. Suppl. 94, 337 (2001) [arXiv: hep-lat/0011075].

[37] D. Becirevic, P. Boucaud, J.P. Leroy, V. Lubicz, G. Martinelli, F. Mescia and F. Rapuano, Phys. Rev. D 60, 074501 (1999) [arXiv: hep-lat/9811003].

[38] CLEO Collaboration, M. Artuso et al., Phys. Rev. Lett. 95, 251801 (2005) [arXiv: hep-ex/0508057.

[39] BABAR Collaboration, B. Aubert et al., Phys. Rev. Lett. 98, 141801 (2007) [arXiv: hep-ex/0607094.

[40] UKQCD Collaboration, K. C. Bowler et al., Nucl. Phys. B 619, 507 (2001) [arXiv: hep-ph/0007020].

[41] CLEO Collaboration, M. Artuso et al., arXiv: 0704.0629 [hep-ex].

[42] CLEO Collaboration, T. K. Pedlar et al., arXiv: 0704.0437 [hep-ex].

[43] V.V. Kiselev, arXiv: hep-ph/021102.

[44] H.G. Dosch, E.M. Ferreira, F.S. Navarra and M. Nielsen, Phys. Rev. D 65, 114002 (2002) [arXiv: hep-ph/0203225.

[45] R.D. Matheus, F.S. Navarra, M. Nielsen and R. Rodrigues da Silva, Phys. Lett. B 541, 265 (2002) [arXiv: hep-ph/0206198. 
[46] M.E. Bracco, M. Chiapparini, F.S. Navarra and M. Nielsen, Phys. Lett. B 605, 326 (2005) [arXiv: hep-ph/0410071.

[47] F.S. Navarra, Marina Nielsen, M.E. Bracco, M. Chiapparini and C.L. Schat, Phys. Lett. B 489, 319 (2000) [arXiv: hep-ph/0005026].

[48] P. Colangelo, G. Nardulli and N. Paver, Z. Phys. C 57, 43 (1993).

[49] K.C. Yang and W.Y.P. Hwang, Z. Phys. C 73, 275 (1997).

[50] A. Khodjamirian and R. Ruckl, Adv. Ser. Direct. High Energy Phys. B 15, 345 (1998) [arXiv: hep-ph/9801443.

[51] UKQCD Collaboration, D.R. Burford et al., Nucl. Phys. B 447, 425 (1995) [arXiv: hep-lat/9503002].

[52] Y.H. Chen, H.Y. Cheng, B. Tseng and K.C. Yang, Phys. Rev. D 60, 094014 (1999) [arXiv: hep-ph/9903453.

[53] N. Isgur D. Scora, B. Grinstein and M.B. Wise, Phys. Rev. D 39, 799 (1989).

[54] V.V. Kiselev and A.V. Tkabladze, Phys. Rev. D 48, 5208 (1993).

[55] Y.M. Wang, et al., in preparation.

[56] L.D. Landau, Nucl. Phys. B 13, 181 (1959).

[57] For a review, see T.J. Eden, P.V. Landshoff, D.I. Olive and J.C. Polkinghorne, "The Analytic S-Matrix", (Cambridge University Press, Cambridge, England, 1966).

[58] L.J. Reinders, H. Rubinstein and S. Yazaki, Phys. Rep. 127, 1 (1985).

[59] L.J. Reinders, H.R. Rubinstein, S. Yazaki, Phys. Lett. B 97, 257 (1980); B 100, 519 (1981)(E).

[60] V.M. Belyaev, V.M. Braun, A. Khodjamirian and R. Rückl, Phys. Rev. D 51, 6177 (1994) [arXiv: hep-ph/9410280.

[61] I. Bediaga and M. Nielsen, Phys. Rev. D 68, 036001 (2003) [arXiv: hep-ph/0304193.

[62] L.J. Reinders, S. Yazaki and H.R. Rubinstein, Phys. Lett. B 103, 63 (1981). 